\title{
On the convergence and scaling of high- order statistical moments in turbulent pipe flow using direct numerical simulations $\odot$ (c)
}

Cite as: Phys. Fluids 29, 125105 (2017); https://doi.org/10.1063/1.4996882

Submitted: 19 July 2017 . Accepted: 23 November 2017 . Published Online: 21 December 2017

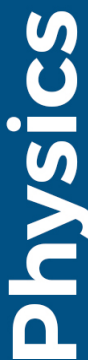

C. Bauer, (D) D. Feldmann, and C. Wagner

\section{COLLECTIONS}

F This paper was selected as Featured

SCI This paper was selected as Scilight
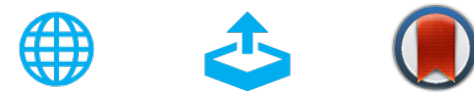

\section{ARTICLES YOU MAY BE INTERESTED IN}

The culmination of an inverse cascade: Mean flow and fluctuations

Physics of Fluids 29, 125102 (2017); https://doi.org/10.1063/1.4985998

Wall-bounded turbulent flows at high Reynolds numbers: Recent advances and key issues

Physics of Fluids 22, 065103 (2010); https://doi.org/10.1063/1.3453711

Scaling of the velocity fluctuations in turbulent channels up to $\mathrm{Re}_{\tau}=2003$

Physics of Fluids 18, 011702 (2006); https://doi.org/10.1063/1.2162185

\section{AIP Advances Fluids and Plasmas Collection}

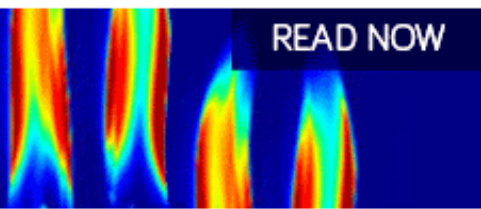




\title{
On the convergence and scaling of high-order statistical moments in turbulent pipe flow using direct numerical simulations
}

\author{
C. Bauer, ${ }^{1,2, a)}$ D. Feldmann, ${ }^{3}$ and C. Wagner ${ }^{1,2}$ \\ ${ }^{1}$ Institute of Aerodynamics and Flow Technology, German Aerospace Center, Bunsenstraße 10, \\ 37073 Göttingen, Germany \\ ${ }^{2}$ Institute of Thermodynamics and Fluid Mechanics, Technische Universität Ilmenau, Helmholtzring 1, \\ 98693 Ilmenau, Germany \\ ${ }^{3}$ Center of Applied Space Technology and Microgravity (ZARM), University of Bremen, Am Fallturm 2, \\ 28359 Bremen, Germany
}

(Received 19 July 2017; accepted 23 November 2017; published online 21 December 2017)

\begin{abstract}
Direct numerical simulations of turbulent pipe flow in a flow domain of length $L=42 R$, friction Reynolds number in the range of $180 \leq R e_{\tau} \leq 1500$, and two different wall-normal grid refinements were carried out and investigated in terms of high-order turbulence statistics. The phenomenology of large local wall-normal velocity fluctuations (velocity spikes) was discussed by means of time series and instantaneous flow-field realisations. Due to their rare appearance both in space and time, statistical high-order moments take a long time to converge. A convergence study was performed and for fully converged statistics the sensitivity of the grid resolution on the wall-normal kurtosis component value at the wall as well as the scaling behaviour of high-order statistics was investigated. The streamwise Reynolds stress as well as the streamwise skewness and the wall-normal flatness exhibited logarithmic Reynolds number dependencies in the vicinity of the wall and scaling laws were derived accordingly. In the bulk flow region, a sudden increase in magnitude in both the streamwise Reynolds stress and skewness was determined for the largest Reynolds number $R e_{\tau}=1500$, while the profiles collapsed well in wall units for $R e_{\tau} \leq 720$. Both Reynolds number dependencies in the near-wall and the bulk region could be related to large-scale outer-flow motions penetrating the buffer layer. While wavelengths related to larger-scale motions $\left(\lambda_{z} \approx 3 R\right)$ were computed for Reynolds numbers up to $R e_{\tau}=720$ by means of two-dimensional two-point velocity correlations, even larger wavelengths related to very-large-scale motions appeared for $R e_{\tau}=1500$. They are probably the reason for the sudden increase in magnitude of streamwise Reynolds stress and skewness, respectively. With the aid of instantaneous flow-field realisations and conditional averaged statistics, the Reynolds dependency of the wall-normal flatness value at the wall was related to the scaling failure of the streamwise Reynolds stress peak. For the lowest Reynolds number $\left(R e_{\tau}=180\right)$, discrepancies between plane channel and pipe flow were found and discussed. Published by AIP Publishing. https://doi.org/10.1063/1.4996882
\end{abstract}

\section{INTRODUCTION}

Turbulent pipe flows have been extensively studied over the last decades by means of both experiments and numerical simulations. While there is a wide overall agreement in terms of turbulent statistics, a discrepancy remains between the kurtosis level of wall-normal velocity fluctuation close to the wall found in experiments and the kurtosis level obtained from direct numerical simulation (DNS). A study of Xu et al. ${ }^{1}$ related the high-kurtosis level of wall-normal velocity fluctuations found in DNS to very strong events (velocity spikes) that are rare both in time and space. Xu et al. performed a DNS of the turbulent plane channel flow of $R e_{\tau}=172$ within a domain of $L_{x}=4 \pi h \times L_{y}=2 \pi h$, where $x, y$, and $h$ represent the streamwise coordinate, the spanwise coordinate, and the channel half height. By comparing numerical results with detailed near-wall laser Doppler anemometry (LDA) measurements, ${ }^{2}$ they concluded that the above-mentioned high-kurtosis events

a)Electronic mail: christian.bauer@dlr.de are usually lacking in experimental data. Besides the restricted access of optical measuring methods to the wall, low measuring time resolutions and limited time series, where extreme events are treated as outliers, were found to be responsible for relatively low wall-normal kurtosis values at the wall. den Toonder and Nieuwstadt ${ }^{3}$ measured higher kurtosis values of $F\left(u_{r}\right) \approx 20$ for intermediate Reynolds numbers of $\operatorname{Re}_{\tau}=520$ and $R e_{\tau}=690$. However, they observed scattering with respect to their low Reynolds number experiments $\left(R e_{\tau}=169\right.$, $\left.R e_{\tau}=315\right)$, where the wall-normal flatness value did not exceed values of $F\left(u_{r}\right)=8$. Additionally, their access to the wall was limited to wall distances of $y^{+} \approx 5$. In this study, we are going to revisit high-order statistical moments for turbulent pipe flow using modern numerical methods and up-to-date computational resources. Open questions with regard to higher statistical moments in wall-bounded turbulence will also be addressed: First, the analysis of Xu et al. exhibited a kurtosis level of $F(w)=25$ at the wall. The turbulent pipe-flow simulations of Eggels et al. ${ }^{4}$ which were restricted to secondorder accuracy in space, reported a somewhat lower value of $F\left(u_{r}\right)=19$ for $R e_{\tau}=180$. Wagner and Friedrich ${ }^{5}$ reported 
a value of $F\left(u_{r}\right)=30$ for the same Reynolds number, computed with second-order accuracy as well. A more recent study by Shishkina and Wagner, ${ }^{6}$ involving a fourth-order finite-volume scheme, showed values between $F\left(u_{r}\right)=27$ and $F\left(u_{r}\right)=31$ at the wall, depending on the grid resolution. Boersma ${ }^{7}$ reported values between $F\left(u_{r}\right)=31$ for $R e_{\tau}=688$ and $F\left(u_{r}\right)=38$ for $R e_{\tau}=1842$, computed with a pseudo-spectral simulation code. Besides the dependency on the grid resolution, the near-wall kurtosis value is likely to converge very slowly, since it corresponds to both spatially and temporarily rare events. In order to investigate the dependency of converged higher statistical moments on the grid resolution, we first carry out a convergence study on a coarser grid, which is still fine in terms of commonly accepted values for DNS. Subsequently, a simulation on a finer grid is performed and the results are integrated over the estimated convergence time. Besides the absolute kurtosis value, the behaviour of the curve approaching the wall is investigated.

In terms of turbulent plane channel flow, the study of Lenaers et al. ${ }^{8}$ investigated the dependency of extreme wall-normal velocity fluctuations on the Reynolds number for Reynolds numbers $180 \leq R e_{\tau} \leq 1000$ involving a pseudo-spectral simulation scheme. Wall-normal kurtosis values between $F(v)=27$ for $R e_{\tau}=180$ and $F(v)=43$ for $R e_{\tau}=1000$ were reported for the plane channel flow. The strong Reynolds number dependency was related to the modulation of small-scale structures near the wall by large-scale motions (LSMs) of the outer layer. ${ }^{9}$ By computing the scaledecomposed velocity skewness, Mathis et al. ${ }^{10}$ showed that the streamwise skewness component is strongly influenced by the amplitude modulation effect for turbulent boundary-layer flow.

In order to resolve large velocity gradients near the wall, a fine grid resolution is required. For the investigation of the influence of the grid refinement, three different flow cases are simulated involving different grid refinements $(P 180 C, P 180$, and $P 180 F$ ), as depicted in Table I. Studies of Chin et al. $^{11}$ and Feldmann et al. ${ }^{12}$ showed the need for long flow domains in the Reynolds number regime of this work to fully capture the physically relevant scales in the near-wall region. Their results imply that a pipe domain length of $L=42 R$ was sufficiently large.

Furthermore, in order to elucidate the scaling behaviour of higher statistical moments in turbulent pipe flow, higher Reynolds number simulations involving friction Reynolds numbers of $R e_{\tau}=360, R e_{\tau}=720$, and $R e_{\tau}=1500$ are carried out (Table I), expanding the scope of previous studies by Wagner et al. ${ }^{5,13,14}\left(R e_{\tau} \leq 320\right)$ and Feldmann and Wagner ${ }^{15}$ $\left(R e_{\tau}=720\right)$. The dynamics of the so-called very-large-scale motion (VLSM) have become important within the last years, since they are known to contribute to Reynolds stresses ${ }^{16,17}$ of high Reynolds number flow $\left(R e_{\tau}>1000\right)$. However, their influence on even higher statistical moments, such as skewness and kurtosis, has not been rigorously studied yet and the question whether and how they act on the scaling of the latter will be addressed in this study.

The aim of the current study is to revise statistical moments in turbulent pipe flow, such as turbulent intensities, skewness, and flatness factors in large domains with high
TABLE I. Turbulent pipe-flow simulation cases. $R e_{\tau}=u_{\tau} R / v$ and $R e_{b}=u_{b} D / v$ are the friction and bulk Reynolds number, where $u_{\tau}$ is the friction and $u_{b}$ is the bulk velocity $(D=2 R) . N_{z}, N_{\varphi}$, and $N_{r}$ are the number of grid points with respect to the axial, azimuthal, and radial direction, respectively. $\Delta z^{+}$, streamwise grid spacing; $R^{+} \Delta \varphi$, azimuthal grid spacing at the wall; $\Delta r_{\min }^{+}$and $\Delta r_{\max }^{+}$, minimal and maximal radial grid spacing, respectively, all grid spacings normalised by wall units. $\Delta t$, averaging interval for statistics; $\Delta t_{b}=\Delta t u_{b} / R$, in bulk time units; $\Delta t^{+}=\Delta t u_{\tau}^{2} / v$, in viscous time units; $\Delta \tau^{+}=\Delta t^{+} L^{+} 2 \pi r^{+}$, averaging coordinate in viscous units. Pipe length for all cases: $L / R=42$.

\begin{tabular}{lcccccc}
\hline \hline Case & $P 180 C$ & $P 180$ & $P 180 F$ & $P 360$ & $P 720$ & $P 1500$ \\
\hline$R e_{\tau}$ & 180 & 180 & 180 & 360 & 720 & 1500 \\
$R e_{b}$ & 5258 & 5258 & 5258 & 11664 & 25992 & 60075 \\
$L / R$ & 42 & 42 & 42 & 42 & 42 & 42 \\
$N_{z}$ & 1536 & 1536 & 1536 & 3072 & 4608 & 8192 \\
$N_{\varphi}$ & 256 & 256 & 256 & 512 & 1024 & 2048 \\
$N_{r}$ & 76 & 84 & 104 & 160 & 222 & 408 \\
$\Delta z^{+}$ & 4.9 & 4.9 & 4.9 & 4.9 & 6.6 & 7.7 \\
$R^{+} \Delta \varphi$ & 4.4 & 4.4 & 4.4 & 4.4 & 4.4 & 4.6 \\
$\Delta r_{\min }^{+}$ & 0.50 & 0.31 & 0.11 & 0.39 & 0.49 & 0.49 \\
$\Delta r_{\max }^{+}$ & 4.4 & 4.4 & 4.4 & 4.4 & 6.6 & 7.8 \\
$\Delta t_{b}$ & 9826 & 28344 & 6682 & 2708 & 788 & 65 \\
$\Delta t^{+}$ & 121050 & 349250 & 82330 & 60200 & 31450 & 4890 \\
$\Delta \tau^{+} \times 10^{12}$ & 1.04 & 2.99 & 0.70 & 2.06 & 4.30 & 2.90 \\
\hline \hline
\end{tabular}

near-wall grid resolutions. The study is also intended to reveal the influence of outer-flow motions penetrating the buffer layer on turbulent statistics and to derive the corresponding laws for higher statistical moments. The results are compared to existing boundary-layer ${ }^{10}$ and turbulent plane channel ${ }^{8}$ studies, extending the scope to Reynolds numbers of $R e_{\tau}=1500$ with respect to the latter study, where VLSM is believed to play a more significant role.

\section{FLOW GEOMETRY AND NUMERICAL METHODOLOGY}

The governing equations in the case of a pressure-driven incompressible flow of a Newtonian fluid in a smooth pipe are the incompressible Navier-Stokes equations in the following dimensionless form:

$$
\begin{gathered}
\frac{\partial \vec{u}}{\partial t}+\vec{u} \cdot \nabla \vec{u}+\nabla p=\frac{1}{R e_{\tau}} \nabla^{2} \vec{u}, \\
\nabla \cdot \vec{u}=0,
\end{gathered}
$$

where $R e_{\tau}=u_{\tau} R / v$ is the friction Reynolds number, based on friction velocity, pipe radius, and kinematic viscosity. Equation (1) is integrated in time using a leapfrog-Euler scheme after being discretised by means of a fourth-order finite-volume method. ${ }^{6,15}$ The scheme is based on a projection method $^{18}$ and consists of three basic steps. First, an auxiliary velocity field is computed from the momentum equation (1) neglecting the pressure term. Then, a Poisson equation is solved in order to fulfil the divergence-free condition before the actual velocity and pressure fields are updated from the auxiliary field and the solution of the Poisson equation. The flow geometry, which is an annular smooth pipe with length $L$ and radius $R$, as shown in Fig. 1, is discretised via staggered grids in a cylindrical coordinate system, where $z$ is the 


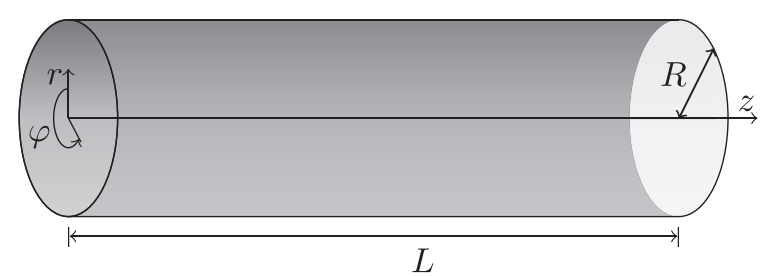

FIG. 1. Pipe geometry involving a cylindrical coordinate system, where $z$ is the axial, $\varphi$ is the azimuthal, and $r$ is the radial coordinate. $L$ is the pipe length and $R$ is the pipe radius.

axial, $\varphi$ is the azimuthal, and $r$ is the radial coordinate. The wall-normal coordinate pointing towards the pipe axis is hereinafter denoted as $y=(R-r)$ and in wall units as $y^{+}=y / \delta_{v}$, where $\delta_{v}=v / u_{\tau}$ is the viscous length scale.

Due to the discretisation, an explicit time integration scheme would require very small time steps in the vicinity of the cylindrical axis in order to meet the stability requirements of the purely explicit scheme. To overcome this problem, a semi-implicit scheme according to Shishkina and Wagner ${ }^{6}$ is applied to the azimuthal component of Eq. (1) only in a narrow region around the cylinder axis. Based on a coarse grid simulation, a convergence criterion for higher statistical moments will be introduced and a simulation performed on a finer grid will be integrated in time until turbulent statistics reach a fully converged state. Table I shows the current simulation setups used within this study. The grid resolution in wall units is set to values of $\Delta z^{+}<8, R^{+} \Delta \varphi<5, \Delta r_{\max }^{+}<8$, and $\Delta r_{\text {min }}^{+}<0.5$ to resolve all relevant turbulent scales. ${ }^{15}$

Statistics are computed by taking advantage of the flow homogeneity with respect to both streamwise and azimuthal directions. Henceforth, the angle brackets indicate an average in space and time as follows:

$$
\langle u\rangle(r)=\frac{1}{L} \frac{1}{2 \pi r} \frac{1}{\Delta t} \int_{t=t_{0}}^{t_{0}+\Delta t} \int_{z=0}^{L} \int_{\varphi=0}^{2 \pi} u(z, \varphi, r, t) r d \varphi d z d t,
$$

where $\Delta t$ is the averaging interval in time. Since this work focusses on the convergence of near-wall statistics, averaging intervals both in space and time are normalised by viscous length scales and viscous time units, respectively. In order to obtain comparable results for different Reynolds numbers and domain lengths, we assume the following averaging coordinate, taking both temporal and spatial averaging intervals in wall units into account,

$$
\Delta \tau^{+}=\Delta t^{+} L^{+} 2 \pi r^{+},
$$

where $\Delta t^{+}=\Delta t u_{\tau}{ }^{2} / v$ is the time interval normalised in viscous time units and $L^{+}$and $2 \pi r^{+}$are the spatial intervals normalised by the viscous length scale. High-order statistical moments of wall-bounded turbulence, defined as follows:

$$
\begin{aligned}
& S\left(u_{i}\right)=\frac{\left\langle u_{i}^{\prime} u_{i}^{\prime} u_{i}^{\prime}\right\rangle}{\left\langle u_{i}^{\prime} u_{i}^{\prime}\right\rangle^{3 / 2},} \\
& F\left(u_{i}\right)=\frac{\left\langle u_{i}^{\prime} u_{i}^{\prime} u_{i}^{\prime} u_{i}^{\prime}\right\rangle}{\left\langle u_{i}^{\prime} u_{i}^{\prime}\right\rangle^{2}}, i \in\{z, \varphi, r\},
\end{aligned}
$$

are denoted as skewness (4) and flatness (5) of a velocity component $u_{i}$. The prime superscript is used to denote the velocity fluctuation of a component with respect to its mean $\left(u_{i}^{\prime}=u_{i}-\left\langle u_{i}\right\rangle\right)$, also referred to as the Reynolds decomposition. The variance of a velocity component $\left\langle u_{i}^{\prime} u_{i}^{\prime}\right\rangle$ is denoted as normal Reynolds stress and its square root as the velocity component's root mean square (rms) value.

\section{CONVERGENCE OF HIGH-ORDER STATISTICAL MOMENTS}

The probability density function $(p d f)$ of the wall-normal velocity component $u_{r}$ at six different distances from the wall is plotted in Fig. 2 and reveals a non-Gaussian behaviour. As a characteristic feature of wall-bounded turbulence, the distribution shifts from right-skewed to left-skewed and finally to right-skewed again with increasing distance from the wall. Simultaneously, the flatness decreases monotonically and converges towards a constant value.

Large wall-normal velocity values with respect to the local root mean square contribute to a high flatness level in the immediate vicinity of the wall. Since they appear rarely both in space and time, a long sampling interval and/or a large domain size is needed in order to obtain fully converged turbulent statistics. In the following, the convergence of high-order statistical moments with respect to the spatial and temporal averaging intervals, specifically the wall-normal kurtosis or flatness, will be investigated. Furthermore, the sensitivity of the statistical quantities to the grid refinement will be discussed. In order to evaluate the convergence of higher statistical moments in turbulent pipe flow, we evaluated statistics at a Reynolds number of $R e_{\tau}=180$ involving wall-normal grid sizes at the wall of $\Delta r_{\text {min }}^{+}=0.31$ for a long time $\left(\Delta t^{+} \approx 350000, P 180\right)$. As a convergence criterion we allowed a statistical value to deviate less than one percent from the final value. Two additional DNSs involving a finer grid $(P 180 F)$ and a coarse one $(P 180 C)$ were then carried out. For those DNS, statistics were integrated in time, at least until the convergence criterion obtained from the analysis of the initial DNS was fulfilled. Fully converged statistics obtained in these three DNS were then compared in order to determine whether there is an influence of the near-wall grid refinement on near-wall turbulent statistical quantities.

Note that the mean square relative error of a velocity component $u_{i}$ due to finite sampling periods can be estimated by the expression ${ }^{19}$

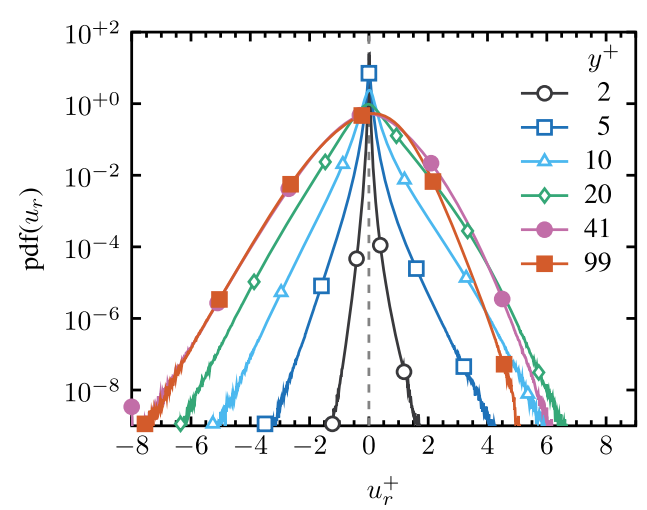

FIG. 2. Probability density functions $(p d f)$ of the wall-normal velocity component $\left(u_{r}\right)$ for $P 180$ at different wall-normal locations $y^{+}$. 


$$
\epsilon^{2}\left(\left\langle u_{i}\right\rangle_{\Delta t}\right)=\overline{\frac{\left(u_{i}-\overline{u_{i}}\right)^{2}}{{\overline{u_{i}}}^{2}}} \frac{2 \mathcal{T}}{\Delta t}
$$

when the integral time scale $\mathcal{T}$ is much smaller than the sampling period $\Delta t .^{20}$ The overbar denotes the true mean, which is obtained by averaging over an infinite time period $\left(\overline{u_{i}}=\int_{0}^{\infty} u_{i} \mathrm{~d} t\right)$ and the angle brackets with subscript $\Delta t$ denote averaging over the finite time period $\Delta t$. The integral time scale is obtained by solving the integral $\mathcal{T}=\int_{0}^{\infty} R_{u_{i} u_{i}}(q) \mathrm{d} q$ with $R_{i i}(q)=\overline{u_{i}(t) u_{i}(t+q)} / \overline{u_{i}(t)^{2}}$, which is the temporal autocorrelation of $u_{i}$. Expanding Eq. (6) by two-dimensional spatial averaging and applying it to the variance $\left(\left\langle u_{i}^{\prime} u_{i}^{\prime}\right\rangle\right)$ and the central fourth-order moment $\left\langle u_{i}^{\prime 4}\right\rangle$ of a velocity component $u_{i}$ give

$$
\begin{aligned}
& \epsilon^{2}\left(\left\langle u_{i}^{\prime} u_{i}^{\prime}\right\rangle\right)=\frac{8 \mathcal{T}_{2}^{(i)} \mathcal{L}_{z 2}^{(i)} \mathcal{L}_{\varphi 2}^{(i)}}{\Delta t_{2} L 2 \pi r}\left(\frac{\overline{u_{i}^{\prime 4}}}{{\overline{u_{i}^{\prime 2}}}^{2}}-1\right), \\
& \epsilon^{2}\left(\left\langle u_{i}^{\prime 4}\right\rangle\right)=\frac{8 \mathcal{T}_{4}^{(i)} \mathcal{L}_{z 4}^{(i)} \mathcal{L}_{\varphi 4}^{(i)}}{\Delta t_{4} L 2 \pi r}\left(\frac{\overline{u_{i}^{\prime 8}}}{{\overline{u_{i}^{\prime \prime}}}^{2}}-1\right),
\end{aligned}
$$

where

$$
\begin{aligned}
& \mathcal{T}_{j}^{(i)}=\int_{0}^{\infty} \frac{\overline{u_{i}^{\prime j}(t) u_{i}^{\prime j}(t+q)}-{\overline{u_{i}^{\prime j}}}^{2}}{\overline{u_{i}^{\prime 2 j}}-{\overline{u_{i}^{\prime j}}}^{2}} \mathrm{~d} q, \\
& \mathcal{L}_{k j}^{(i)}=\int_{0}^{\infty} \frac{\overline{u_{i}^{\prime j}(k) u^{\prime j}(k+\Delta k)}-{\overline{u_{i}^{\prime j}}}^{2}}{\overline{u_{i}^{\prime 2 j}}-{\overline{u_{i}^{\prime j}}}^{2}} \mathrm{~d} \Delta k,
\end{aligned}
$$

with $k=z, \varphi$ are the integral time and length scales of $u_{i}^{\prime j}$, respectively. Note that the prime symbols in Eqs. (7) and (8) denote the fluctuation about the true mean $\left(u_{i}^{\prime}=u_{i}-\overline{u_{i}}\right)$. Inverting Eqs. (7) and (8) and substituting them into Eq. (3) yield an estimate of the spatio-temporal averaging interval needed to obtain a certain relative mean square error,

$$
\begin{aligned}
\Delta \tau_{i, 2}^{+} & =\frac{8 \mathcal{T}_{2}^{(i)+} \mathcal{L}_{z 2}^{(i)+} \mathcal{L}_{\varphi 2}^{(i)+}}{\epsilon^{2}}\left(\frac{\overline{u_{i}^{\prime 4}}}{{\overline{u_{i}^{\prime 2}}}^{2}}-1\right), \\
\Delta \tau_{i, 4}^{+} & =\frac{8 \mathcal{T}_{4}^{(i)+} \mathcal{L}_{z 4}^{(i)+} \mathcal{L}_{\varphi 4}^{(i)+}}{\epsilon^{2}}\left(\frac{\overline{u_{i}^{\prime 8}}}{{\overline{u_{i}^{\prime 2}}}^{2}}-1\right) .
\end{aligned}
$$

Estimating the spatio-temporal averaging intervals needed to obtain a relative error of one percent $(\epsilon=0.01)$, Eqs. (11) and (12) are computed at prominent wall-normal locations for the statistical quantities of interest. Note that the values on the right-hand side of the above equations are estimated involving finite spatio-temporal averaging intervals themselves (i.e., $\left.\overline{u_{i}} \approx\left\langle u_{i}\right\rangle\right)$. Furthermore, integral length and time scales as stated in Eqs. (9) and (10) were approximated by integrating the corresponding autocorrelation functions up to the first zero crossing. ${ }^{21}$ Values of $\Delta \tau_{z, 2}^{+}\left(y^{+}=15\right)=1.1 \cdot 10^{9} \sim \mathcal{O}\left(10^{9}\right)$ and $\Delta \tau_{r, 4}^{+}\left(y^{+}=1\right)=1.2 \cdot 10^{11} \sim \mathcal{O}\left(10^{11}\right)$ are obtained. The large discrepancy between the averaging intervals needed for the quantities above to obtain statistics of the same quality is due to two competing effects. On the one hand, the required averaging time or area increases with increasing integral time or length scales, respectively. While the integral scales of $\left\langle u_{z}^{\prime} u_{z}^{\prime}\right\rangle$ at $y^{+}=15$ equal to $\mathcal{T}_{2}^{(z)+}=8, \mathcal{L}_{z, 2}^{(z)+}=110$, and $\mathcal{L}_{\varphi, 2}^{(z)+}=13$, the scales of $\left\langle u_{r}^{\prime 4}\right\rangle$ at $y^{+}=1$ are much smaller $\left(\mathcal{T}_{4}^{(r)+}=1.2, \mathcal{L}_{z, 4}^{(r)+}=11.5\right.$, and $\left.\mathcal{L}_{\varphi, 4}^{(r)+}=6.8\right)$. On the other hand, high-order moments appear in the right-hand side of Eqs. (11) and (12). Higher order terms exhibit larger values $\left(\left.F\left(u_{z}\right)\right|_{y^{+}=15}=2.2,\left.\left(\left\langle u_{z}^{\prime 8}\right\rangle /\left\langle u_{z}^{\prime 4}\right\rangle^{2}\right)\right|_{y^{+}=15}=4.8\right)$ than lower order terms and the strong intermittent behaviour of the wallnormal velocity at the wall leads to much larger values than the streamwise velocity component $\left(\left.\left(\left\langle u_{z}^{\prime 8}\right\rangle /\left\langle u_{z}^{\prime 4}\right\rangle^{2}\right)\right|_{y^{+}=1}=85\right.$, $\left.\left.\left(\left\langle u_{r}^{\prime 8}\right\rangle /\left\langle u_{r}^{\prime 4}\right\rangle^{2}\right)\right|_{y^{+}=15}=1.7 \cdot 10^{4}\right)$. In summation the second effect weighs more heavily, leading to larger sampling intervals needed for the wall-normal flatness. The aforementioned quantities integrated over different time intervals are shown in Fig. 3 together with the wall-normal skewness component. While the peak of the streamwise Reynolds stress component in Fig. 3(a) already exhibits a value within the one-percent criterion at the first evaluation time $\left(\Delta t^{+}=700, \Delta \tau^{+} \approx 6 \times 10^{9}\right)$, skewness and flatness of the wall-normal velocity take much longer to converge. While skewness stays within the boundaries of the one-percent criterion after being averaged in time over $\Delta t^{+} \approx 30000\left(\Delta \tau^{+} \approx 2.6 \times 10^{11}\right)$, as Fig. 3(b) indicates, for the high-kurtosis value at $y^{+}=1$ this is only the case for $\Delta t^{+}>72500\left[\Delta \tau^{+}>6.2 \times 10^{11}\right.$, Fig. 3(c) $]$. The actual intervals needed for the convergence of the statistics are larger than the ones estimated by Eqs. (11) and (12), though they are of about the same order of magnitude $\left(\mathcal{O}\left(10^{9}\right)\right.$ for streamwise Reynolds stress and $\mathcal{O}\left(10^{11}\right)$ for wall-normal flatness). In order to obtain fully converged near-wall statistics, the flow variables of all Reynolds number cases of this work are accordingly integrated over at least $\Delta \tau^{+}=6.2 \times 10^{11}$.
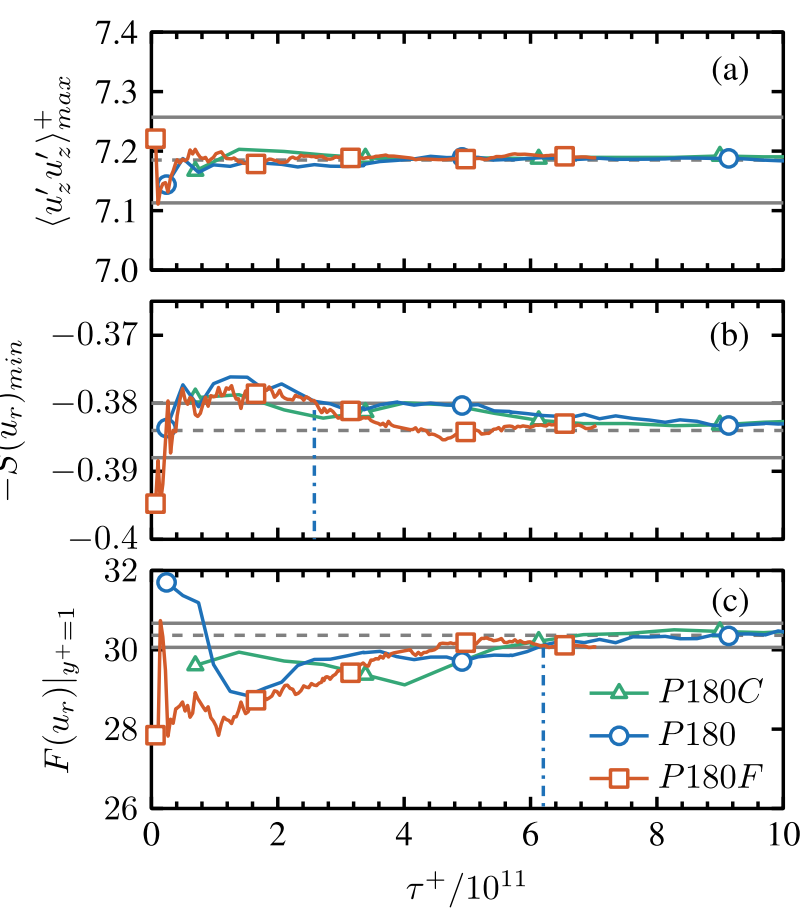

FIG. 3. Convergence of Reynolds stresses and high-order statistical moments for different wall-normal grid spacings. (a) $\left\langle u_{z}^{\prime} u_{z}^{\prime}\right\rangle^{+}$at $y^{+}=15$. (b) $S\left(u_{r}\right)$ at $y^{+}=13.5$. (c) $F\left(u_{r}\right)$ at $y^{+}=1$. 

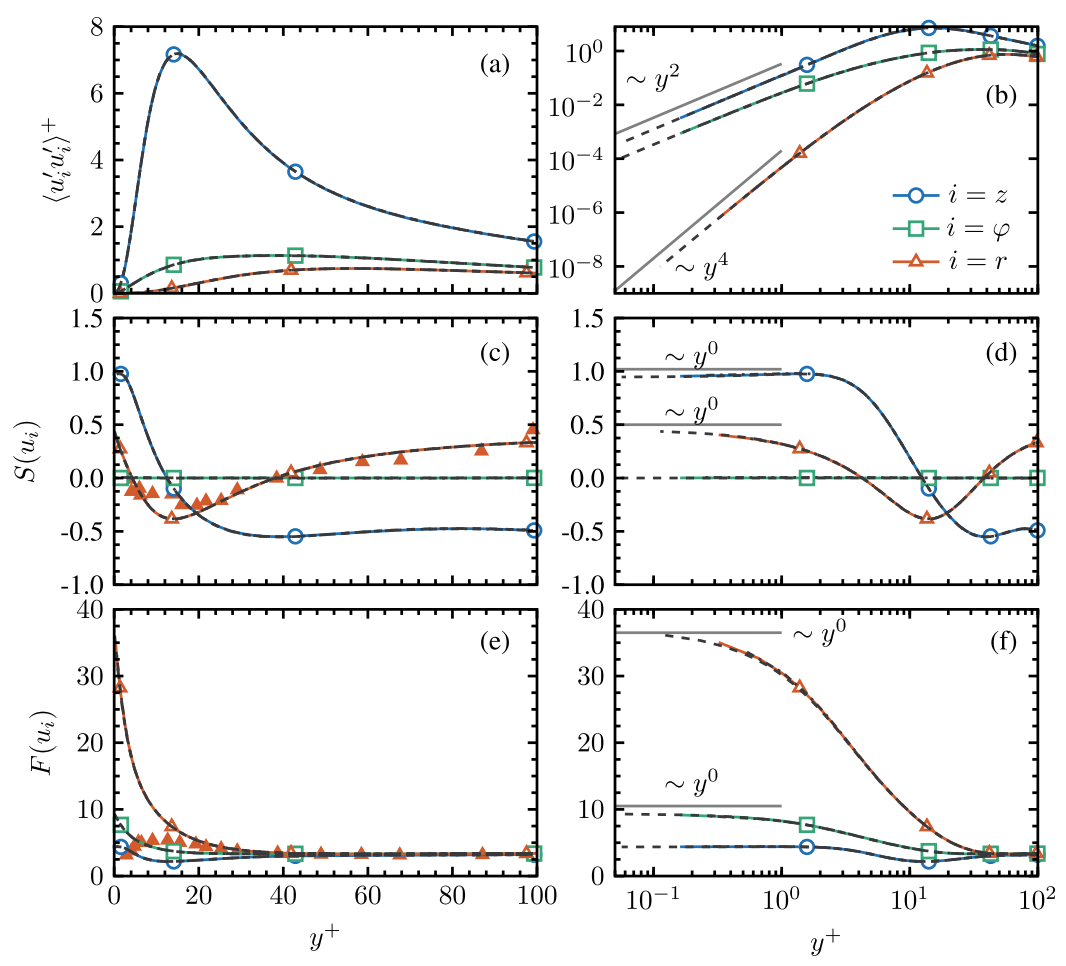

FIG. 4. Reynolds stresses and high-order statistical moments of $P 180$ (solid lines), $P 180 F$ (dashed black lines), and $P 180 C$ (dashed-dotted black lines). Closed orange triangles, LDA data. ${ }^{2}[(a)$ and (b)] Reynolds stresses $\left\langle u_{i}^{\prime} u_{i}^{\prime}\right\rangle^{+}$. [(c) and (d)] Skewness $S\left(u_{i}\right)$. [(e) and (f)] Flatness $F\left(u_{i}\right)$. The right column shows the asymptotic behaviour at the wall.

Fully converged statistics in the vicinity of the wall are compared in Fig. 4. Reynolds stresses [(a) and (b)], skewness [(c) and (d)], and flatness [(e) and (f)] are plotted against the wall distance in wall units shown both in linear and logarithmic coordinates. While the fully converged profiles of Reynolds stresses and skewness of $P 180 F$ and $P 180 C$ collapse nicely, the profile of the wall-normal flatness varies little in the immediate vicinity of the wall, as Fig. 4(e) reveals.

By applying the Taylor expansion to the velocity components at the wall, one can derive the asymptotic limit of the statistical moments as they approach the wall $(r \rightarrow R)$. These values are plotted as dashed-dotted lines in Figs. 4(b), 4(d), and 4(f). The comparison to our data reveals that they match the analytically derived expressions in terms of Reynolds stresses, where

$$
\begin{aligned}
& \lim _{r \rightarrow R}\left\langle u_{z}^{\prime} u_{z}^{\prime}\right\rangle \sim y^{2}, \\
& \lim _{r \rightarrow R}\left\langle u_{\varphi}^{\prime} u_{\varphi}^{\prime}\right\rangle \sim y^{2}, \\
& \lim _{r \rightarrow R}\left\langle u_{r}^{\prime} u_{r}^{\prime}\right\rangle \sim y^{4},
\end{aligned}
$$

quite well. For skewness and flatness all components asymptotically approach a constant value, as they reach the wall. Figure 4(d) indicates that in the case of the coarse grid simulation, a constant value at the wall has not been reached yet. For wall-normal kurtosis, where the gradients close to the wall are much steeper, none of the two simulations reaches a constant value. However the separation between the two curves is rather small, as shown in Fig. 4(f). This leads to the conclusion that in the vicinity of the wall, a grid sizing of $\Delta r_{\text {min }}^{+} \approx 0.3$ is sufficiently small for wall-normal kurtosis to fulfil the boundary condition correctly. For all of our cases, the converged value of wall-normal kurtosis was around 35 and thus much higher than the one obtained from experiments, ${ }^{2}$ as the red triangles in Fig. 4(e) indicate, which has been reported by Xu et al. ${ }^{1}$ and described above.

\section{SCALING OF HIGH-ORDER STATISTICAL MOMENTS}

In order to investigate Reynolds number dependency of high-order statistical moments, turbulent pipe-flow simulations involving four different Reynolds numbers, as listed in Table I, were carried out as well. Statistics were obtained by integrating flow variables in space and time over at least $\Delta \tau^{+}=2.6 \times 10^{11}$, as postulated before. Figure 5 shows Reynolds stress, skewness, and flatness profiles for all Reynolds numbers, plotted over the radial direction normalised in wall (left column) and bulk units (right column).

Reynolds stresses for $R e_{\tau} \in\{180,360,720,1500\}$ are shown in Figs. 5(a) and 5(b). They exhibit a scaling failure when scaled in wall units. They further reveal a shift to higher peak values around $y^{+}=15$ for increasing $R e$ [see Fig. 6(a)]. This well-known effect is also discussed by Hoyas and Jiménez ${ }^{23}$ and Feldmann et al. ${ }^{12}$ amongst others. However, unlike its channel counterpart, the peak value obtained from turbulent pipe flow cannot be fitted with a logarithmic scaling law over the full range of Reynolds numbers. For $R e_{\tau}>360$, the dependency of the $\left\langle u_{z}^{\prime} u_{z}^{\prime}\right\rangle^{+}$peak on $R e_{\tau}$ is fitted by the logarithmic expression

$$
\left\langle u_{z}^{\prime} u_{z}^{\prime}\right\rangle_{\max }^{+}=0.67 \log \left(R e_{\tau}\right)+3.324, \quad R e_{\tau}>360,
$$

indicated by the dashed line in Fig. 6(a), similar to the expression $\left\langle u^{\prime} u^{\prime}\right\rangle_{\max }^{+}=0.642 \log \left(R e_{\tau}\right)+3.66$ derived by Lee and Moser ${ }^{24}$ for plane channel flow.

The velocity skewness profiles for the different Reynolds numbers are shown in Figs. 5(c) and 5(d). In terms of the streamwise skewness, the dependency on the Reynolds 

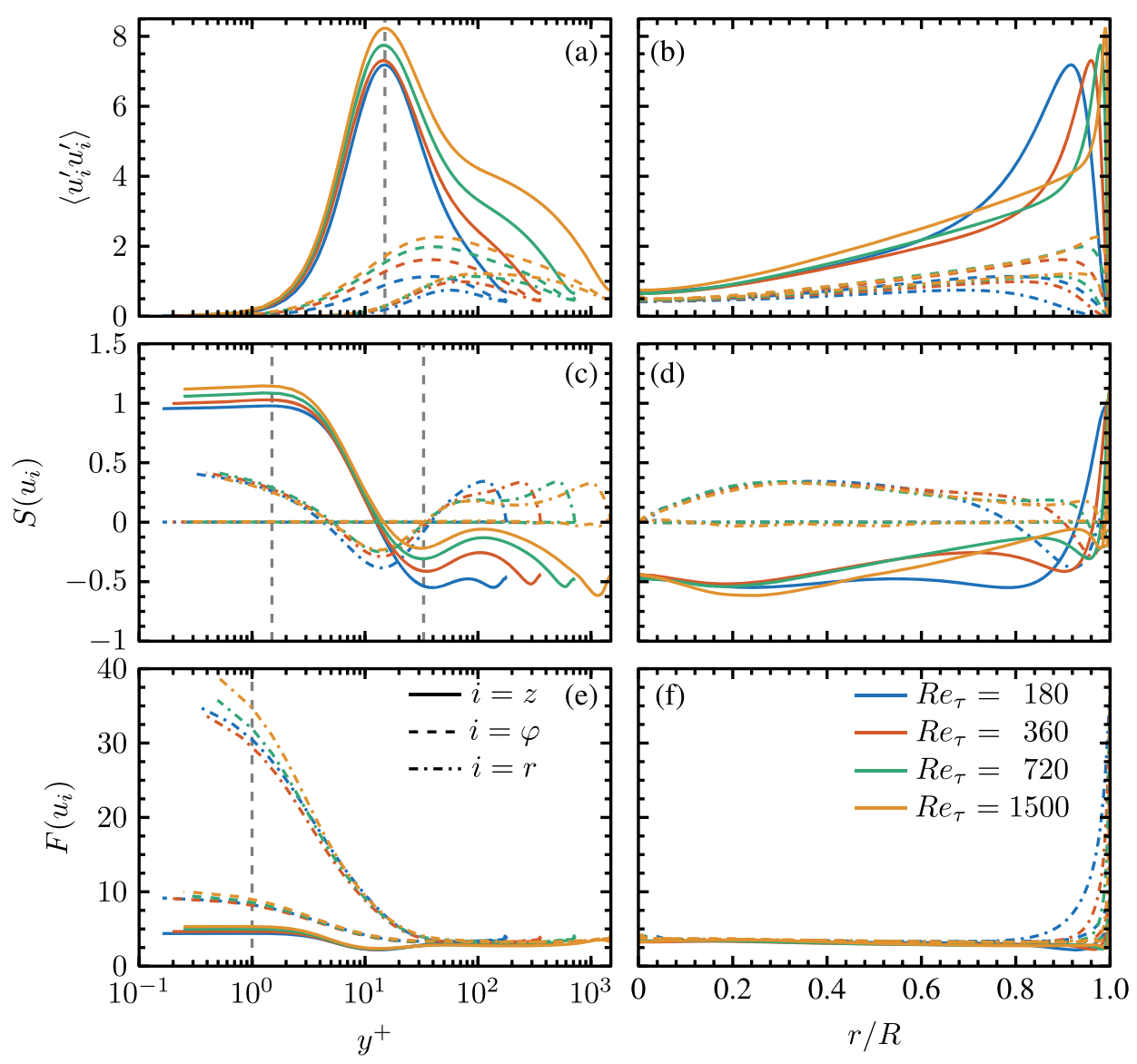

FIG. 5. Scaling of Reynolds stresses and high-order statistical moments. [(a) and (b)] $\left\langle u_{i}^{\prime} u_{i}^{\prime}\right\rangle^{+}$. [(c) and (d)] $S\left(u_{i}\right)$. [(e) and (f)] $F\left(u_{i}\right)$. Blue solid line, $R e_{\tau}=180$; orange solid line, $R e_{\tau}=360$; green solid line, $R e_{\tau}=720$; yellow solid line, $R e_{\tau}=1500$. Solid lines, streamwise component $i=z$; dashed lines, spanwise component $(i=\varphi)$; dasheddotted lines, wall-normal component $i=r$. number is clearly visible in the near-wall region and henceforth investigated at two prominent locations of the profiles, namely, the maximum of the streamwise skewness very close to the wall $\left(y^{+}=1.4\right)$ and the local minimum at a wall distance of approximately $y^{+} \approx 33$. At both the locations, the skewness value shifts towards larger skewness values. This leads to a larger positively skewed streamwise velocity distribution at the wall and a less negatively skewed one at $y^{+} \approx 33$ for higher Reynolds numbers. The Reynolds number dependency of the latter values is fitted by the logarithmic expressions $\left.S\left(u_{z}\right)\right|_{y^{+} \approx 1.4}=0.082 \log \left(R e_{\tau}\right)+0.548$ and $\left.S\left(u_{z}\right)\right|_{y^{+} \approx 33}$ $=0.156 \log \left(R e_{\tau}\right)-1.343$, indicated by dashed lines in Fig. 6(b) together with the corresponding skewness values of the current DNS (circles), pipe-flow DNS data from the work of El Khoury et al. $^{22}$ (triangles), and experimental boundarylayer data of Mathis et al. ${ }^{10}$ at $y^{+} \approx 33$. The last defined logarithmic expression predicts the values of the experimental boundary-layer data fairly well up to $R e_{\tau}=7300$, where the values begin to deviate. While the spanwise skewness exhibits a value of zero over the full flow domain, the wallnormal skewness, indicated by dashed-dotted lines in Figs. 5(c) and 5(d), scales well for $R e_{\tau} \geq 360$ throughout the full flow domain. For $R e_{\tau}=180$, however, there appears to be a low Reynolds number effect, visible for the local minimum value at a wall distance of $y^{+}=13.5$, corresponding to the peak location in streamwise Reynolds stresses and turbulent kinetic energy production. The value of the local minimum equals $S\left(u_{r}\right)=-0.38$ for $R e_{\tau}=180$ and then settles at a value of $S\left(u_{r}\right) \approx-0.25$ for higher Reynolds numbers.
As presented in Fig. 5(f), all flatness components scale well throughout most of the flow domain. Only in the very vicinity of the wall [Fig. 5(e)] does a dependency on the Reynolds numbers in the wall-normal flatness appear in terms of a tendency towards higher values with increasing $R e_{\tau}$. Figure 6(c) shows the wall-normal flatness values at $y^{+}=1$ from the current pipe-flow simulations together with pipe-flow DNS data from the work of Boersma ${ }^{7}$ and plane channel DNS data from the work of Lenaers et al. ${ }^{8}$ While the pipe-flow flatness values at $y^{+}=1$ for $R e_{\tau} \geq 360$ can be fitted by the logarithmic expression $3.7 \log \left(R e_{\tau}\right)+7.7$, indicated by the dashed line in Fig. 6(c), the value for the $R e_{\tau}=180$ does not match the logarithmic law. Furthermore, the discrepancy between the kurtosis value at the wall obtained from turbulent pipe and plane channel flow is largest for the lowest Reynolds number. While the pipe flow at $R e_{\tau}=180$ exhibits a wall-normal flatness value of $F\left(u_{r}\right)=30$ at $y^{+}=1$, the corresponding channel flow simulation, indicated by triangles in Fig. 6(c), exhibits values of $F(v)=23.3$ at the same distance from the wall. The similarity between the scaling failure of the peak values of the streamwise Reynolds stress and the wall-normal flatness component-a logarithmic Reynolds number dependency for $R e_{\tau} \geq 360$ with a tendency towards higher values for larger $R e_{\tau}$-implies that the corresponding coherent flow structures, namely, wall-layer streaks and velocity spikes, are influenced by the bulk flow in a similar manner for higher Reynolds numbers. The interaction of these coherent motions for different Reynolds numbers will be discussed in Sec. V. Note that in the outer-flow region, all components of Reynolds stresses, skewness, and flatness scale 

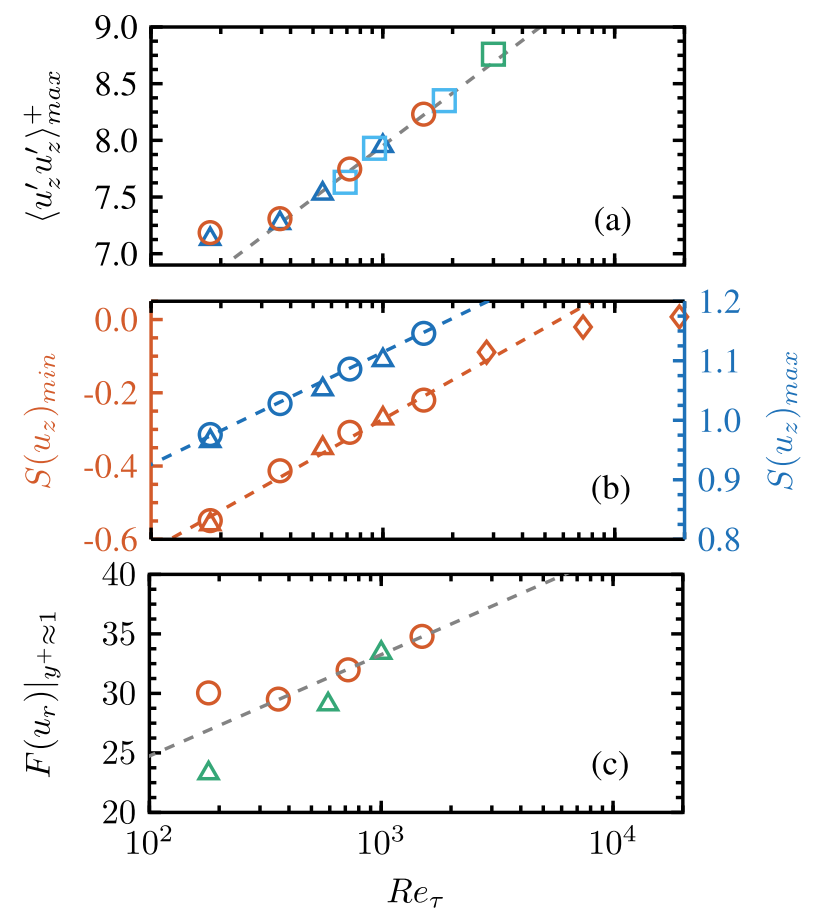

FIG. 6. Scaling failures of minima and maxima in high-order statistics. Extrema locations, see Fig. 5. (a) $\left\langle u_{z}^{\prime} u_{z}^{\prime}\right\rangle_{\max }^{+}$at $y^{+} \approx 15$. (b) $S\left(u_{z}\right)_{\max }$ at $y^{+}=1.4, S\left(u_{z}\right)_{\min }$ at $y^{+} \approx 33$. (c) $F\left(u_{r}\right)$ at $y^{+}=1$. Pipe-flow DNS data: circles, current DNS; (a) blue triangles, El Khoury et al. ${ }^{22}$ light blue squares, Boersma; ${ }^{7}$ green squares, Ahn et al..$^{25}$ (b) Triangles, El Khoury et al. ${ }^{22}$ Experimental boundary-layer data: (b) orange rhombuses, Mathis et al. ${ }^{10}$ Plane channel flow DNS data: (c) green triangles, Lenaers et al. ${ }^{8}$

well with the Reynolds number up to $R e_{\tau}=720$ when plotted over the wall-normal coordinate normalised by the pipe radius [Figs. 5(b), 5(d), and 5(e)]. For $R e_{\tau}=1500$, the streamwise Reynolds stress and skewness profiles exhibit a discrepancy with respect to the other Reynolds number profiles. The sudden increase in $\left\langle u_{z}^{\prime} u_{z}^{\prime}\right\rangle$ for friction Reynolds numbers between 720 and 1500, which was discussed by Feldmann et al., ${ }^{12}$ is often associated with energetically emerging very-large-scale motions. ${ }^{26}$ This connection will be discussed in more detail in Sec. V.

\section{COHERENT STRUCTURES}

Coherent structures play an important role for the distribution of kinetic energy in wall-bounded turbulent flows. Long elongated coherent regions of either high- or low-momentum fluid at a wall distance of $y^{+} \approx 15$, also denoted as walllayer streaks, are well-known phenomena and contribute most to the formation of the $\left\langle u_{z}^{\prime} u_{z}^{\prime}\right\rangle$ peak. ${ }^{27}$ Their spanwise spacing, extracted from two-point velocity correlations or premultiplied energy spectra, exhibits values of $\lambda^{+} \approx 100$ for turbulent plane channel flow ${ }^{28}$ and $\lambda_{\varphi}^{+} \approx 120$ for turbulent pipe flow. ${ }^{5,12,29}$ Regarding the streamwise extension of wall-layer streaks, correlation lengths of $\lambda_{z}^{+} \approx 1000$ have been reported by different studies. ${ }^{13,29}$ The failing of wall scaling of the streamwise Reynolds stress peak, described in Sec. IV, could be related to outer-flow coherent motions that penetrate the buffer layer. ${ }^{23}$ These motions and their impact on the scaling failure of the wall-normal kurtosis component will be described in Sec. V B. Furthermore, local wall-normal fluctuations in the very vicinity of the wall are found to contribute most to the high-kurtosis level at the wall and will be described in the following.

\section{A. Velocity spikes}

$\mathrm{Xu}$ et al. ${ }^{1}$ showed that values contributing most to the high level of wall-normal kurtosis are very-large local wall-normal velocity fluctuations that are rare both in space and time and denoted these events as velocity spikes. Time series of wall-normal velocity fluctuations normalised by the local root mean square value at a wall-normal location of $y^{+}=2$ presented in Fig. 7 exhibit such an event at time $t^{+}=505$. The wall-normal velocity peak, shown in Fig. 7(a), coincides with a positive streamwise fluctuation, visible in Fig. 7(b), leading to a turbulent sweep, as shown in Fig. 7(c).

The same conclusion can be drawn from Fig. 8 where the instantaneous flow field is visualised in planes cutting the probe location of Fig. 7. Very close to the wall, regions of high wall-normal velocity fluctuations always coincide with regions of positive streamwise fluctuations that appear to be footprints of high-velocity streaks. Strongly negative wall-normal velocity regions, on the contrary, are rather located in between regions of low- and high-speed streamwise momentum. Furthermore, Fig. 8 indicates that velocity spikes occur in positive and negative pairs clustering in adjacent regions.

Both Figs. 7 and 8 reveal that velocity spikes are settled in the very vicinity of the wall. The time signal plotted as the blue line in Fig. 7, taken at a probe location of $y^{+}=49.3$, does not exhibit the signature of the spike event.
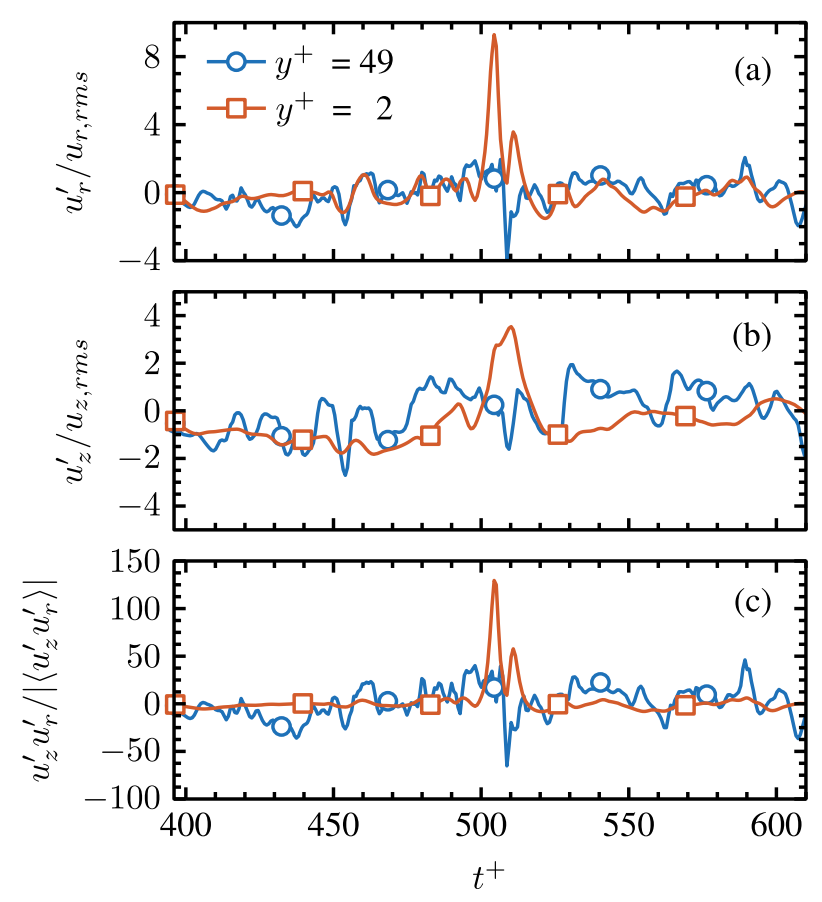

FIG. 7. Time series of $u_{r}^{\prime}(\mathrm{a}), u_{z}^{\prime}(\mathrm{b}), u_{z}^{\prime} u_{r}^{\prime}$ (c), normalised by the corresponding rms value recorded at wall-normal positions $y^{+}=2$ and 49 . 


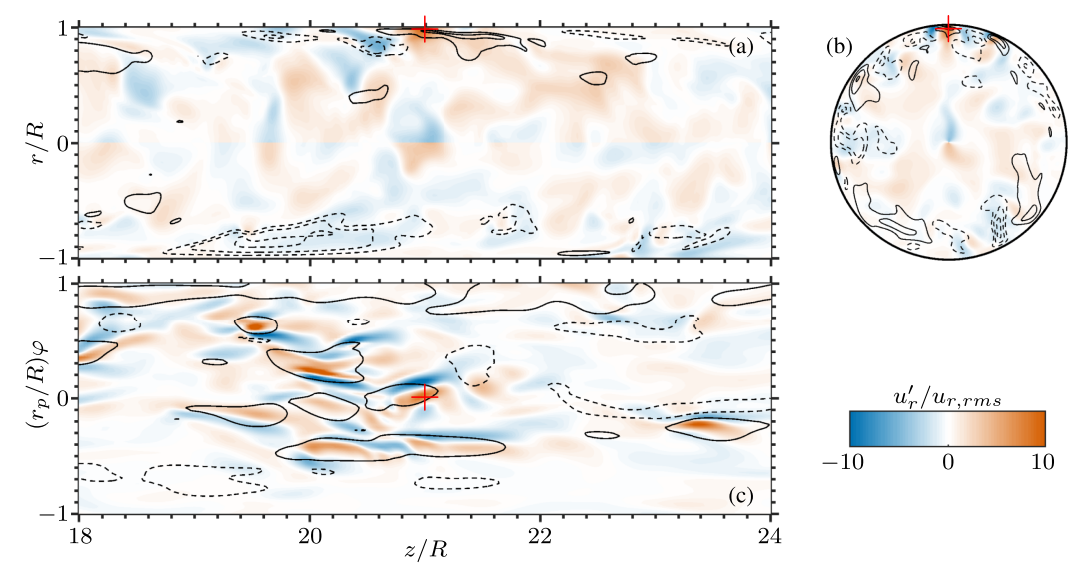

FIG. 8. Instantaneous snapshots of the wall-normal velocity component at $t^{+}=505$ in planes cutting the probe location (red plus) of the time series shown in Fig. 7. Black lines show isocontours of positive (-) and negative (- - ) streamwise velocity fluctuations $u_{z}^{\prime}$ normalised by $u_{\tau}$. [(a) and (b)] Isocontours have an increment of 2 ranging from -6 to 6 . (c) Increment of 0.8 , ranging from -0.8 to 0.8 .

\section{B. Very-large-scale motions}

Amongst others, the study of Kim and Adrian ${ }^{16}$ revealed the so-called very-large-scale motions (VLSMs) occurring in turbulent pipe flow. In contradiction to earlier studies, these structures were found to contribute to the turbulent kinetic energy and are thus important for the understanding of turbulent wall-bounded flows. ${ }^{16,31-37}$ Experimental pipe flow studies by Kim and Adrian, ${ }^{16}$ Guala et al. ${ }^{33}$ and Monty et al. ${ }^{17,38}$ measured typical wavelengths related to VLSM ranging from $10 R<\lambda_{z}<14 R$ up to $14 R<\lambda_{z}<25 R$, depending on the study. Length scales corresponding to the energycontaining motions can be extracted from pre-multiplied energy spectra. ${ }^{16,26,31,39-41}$

The peak wavelength in pre-multiplied energy spectra corresponds to the size of the most energy-containing motions. In the case of wall-layer streaks at $y^{+}=15$, the stream- and spanwise wavelengths yield $\lambda_{z}^{+} \approx 1000$ and $\lambda_{\varphi}^{+} \approx 120$, as reported elsewhere. ${ }^{12}$ In the vicinity of the wall, the imprint of the outer-flow large-scale motions is visible as a local large wavelength peak at high Reynolds number flows, where the scales are sufficiently separated. The interaction of large-scale outer-flow motions is also visible through instantaneous flowfield realisations. Figure 9 shows isocontours of streamwise velocity fluctuations in wall-parallel pipe segments of a constant spatial extension in wall units. At a wall distance of $y^{+}=15$ for $R e_{\tau}=180$ homogeneously distributed high- and low-velocity streaks dominate the picture; see Fig. 9(a). For intermediate $R e_{\tau} \in\{360,720\}$, the streaks begin to organise in clusters at the same wall-normal distance; see Figs. 9(b) and 9(c). However, these clusters do not reach the full length normally associated with VLSM. For $R e_{\tau}=1500$, streaks coherently align to groups of overall streamwise extent comparable to VLSM found in the outer-flow region, shown at a wall distance of $y^{+}=300$ for $\operatorname{Re}_{\tau}=1500$ in Fig. 9(e). The spacing of the larger coherent regions in the near-wall
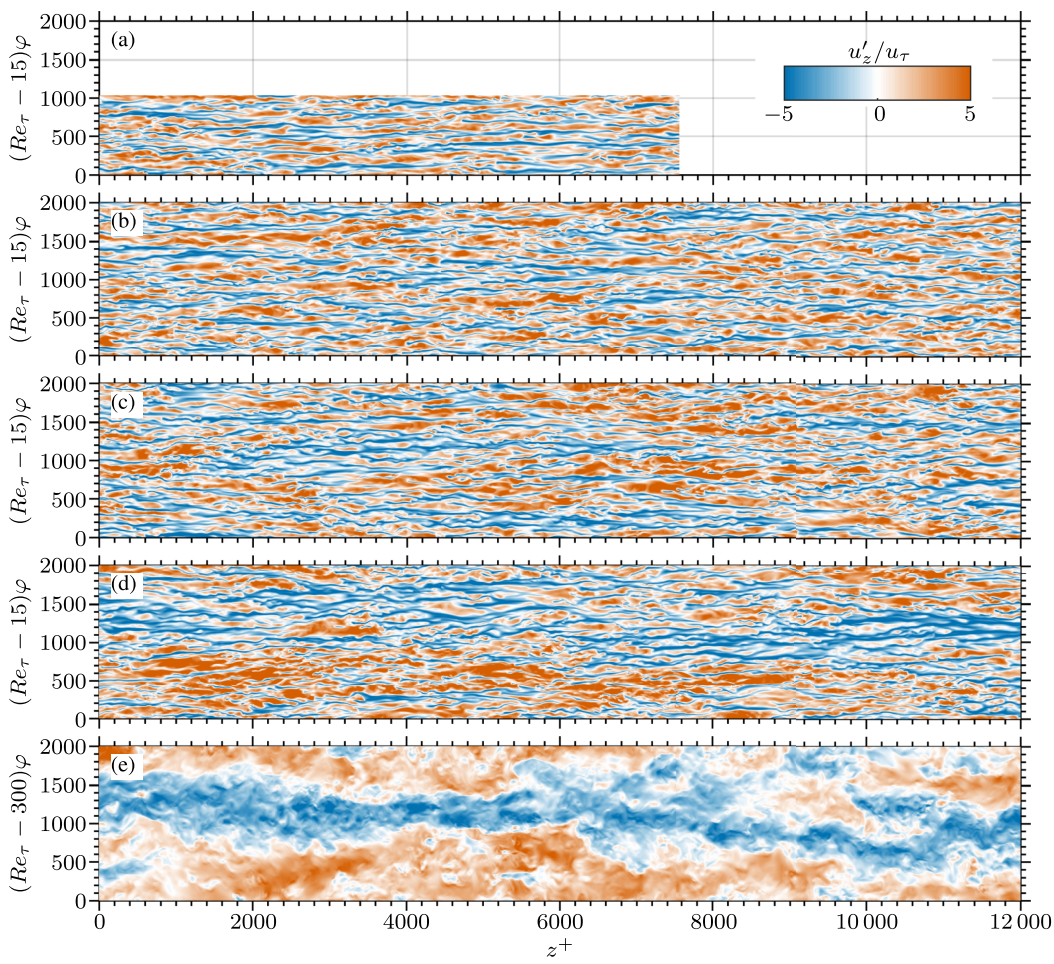

FIG. 9. Isocontours of streamwise velocity fluctuations in a wall-parallel pipe segment of size $l_{z}^{+} \times l_{\varphi}^{+}=12000 \times$ 2000. [(a)-(d)] $y^{+}=15$. (e) $y^{+}=300$. (a) $R e_{\tau}=180$. (b) $R e_{\tau}=360$. (c) $R e_{\tau}=720$. [(d) and (e) $R e_{\tau}=1500$. 
region $\left(y^{+}=15\right)$ corresponds to the local large wavelength peak in the spanwise pre-multiplied spectrum, which is related to the global peak in the spectrum of the bulk region. ${ }^{12}$

To estimate the spatial extension of coherent structures in the near-wall and in the bulk region with respect to all three dimensions, two-dimensional two-point velocity correlations with reference points at $y^{+}=15$ and $r / R=0.6$ in three orthogonal planes, where

$$
\begin{aligned}
& R_{z z}(\Delta z, \Delta r, r)=\frac{\left\langle u_{z}^{\prime}(0, r) u_{z}^{\prime}(\Delta z, r+\Delta r)\right\rangle}{u_{z, r m s}^{\prime 2}(r)}, \\
& R_{z z}(\Delta z, \Delta \varphi, r)=\frac{\left\langle u_{z}^{\prime}(0,0, r) u_{z}^{\prime}(\Delta z, \Delta \varphi, r)\right\rangle}{u_{z, r m s}^{\prime 2}(r)}, \\
& R_{z z}(\Delta \varphi, \Delta r, r)=\frac{\left\langle u_{z}^{\prime}(0, r) u_{z}^{\prime}(\Delta \varphi, r+\Delta r)\right\rangle}{u_{z, r m s}^{\prime 2}(r)}
\end{aligned}
$$

are shown in Fig. 10. The values of the contour lines are chosen to exhibit values of $R_{z z}=0.62$ and $R_{z z}=0.12$, where the latter value indicates the same spatial extension as the pre-multiplied spectra in terms of wall-layer streaks. Figures 10(a) and 10(b) show the two-dimensional velocity correlations of the streamwise velocity component for the different Reynolds numbers at wall distances of $y^{+}=15$ [Fig. 10(a)] and $r / R=0.6$ [Fig. 10(b)]. In Figs. 10(c) and 10(d), the same quantities are shown in an $r z$-plane with reference points at $y^{+}=15$ [Fig. 10(c)] and $r / R=0.6$ [Fig. 10(d)]. Finally, Figs. 10(e) and 10(f) present the velocity correlations in the pipe cross section, involving the same reference points of $y^{+}=15$ [Fig. $\left.10(\mathrm{e})\right]$ and $r / R=0.6$ [Fig. 10(f)]. From the above-shown correlation functions, the average spatial extension of coherent structures can be estimated. Figures 10(a), 10(c), and 10(e) indicate, in agreement with the literature, ${ }^{5,28,30}$ the extension of the wall-layer streaks for $R e_{\tau}<360$,

$$
l_{z, \text { streak }}^{+} \times l_{\varphi, \text { streak }}^{+} \times l_{r, \text { streak }}^{+} \approx 1000 \times 50 \times 50 .
$$

For higher Reynolds numbers, highlighted by the green and yellow contour lines in Figs. 10(a), 10(c), and 10(e), the walllayer streaks correlate with larger regions of coherent momentum caused by the streak packaging shown in Fig. 9, which leads to an overestimation of their streamwise extension by the two-dimensional velocity correlations. While wall-layer streaks scale well in wall units, the outer-flow large-scale motions scale in bulk units; see Figs. 10(b), 10(d), and 10(f). The spanwise spacing extracted from Figs. 10(b) and 10(f) of large-scale motions (LSMs) is $\lambda_{\varphi} / R \approx R$ and the average streamwise extension $\lambda_{z} / R \approx 3 R$ for $R e_{\tau}<720$ [Figs. 10(b) and $10(\mathrm{~d})]$. For higher Reynolds numbers, the average streamwise extension is found to be much longer, as the yellow contour lines in Figs. 10(b) and 10(d) indicate, and related to VLSM appearing at such high Reynolds numbers. The overall
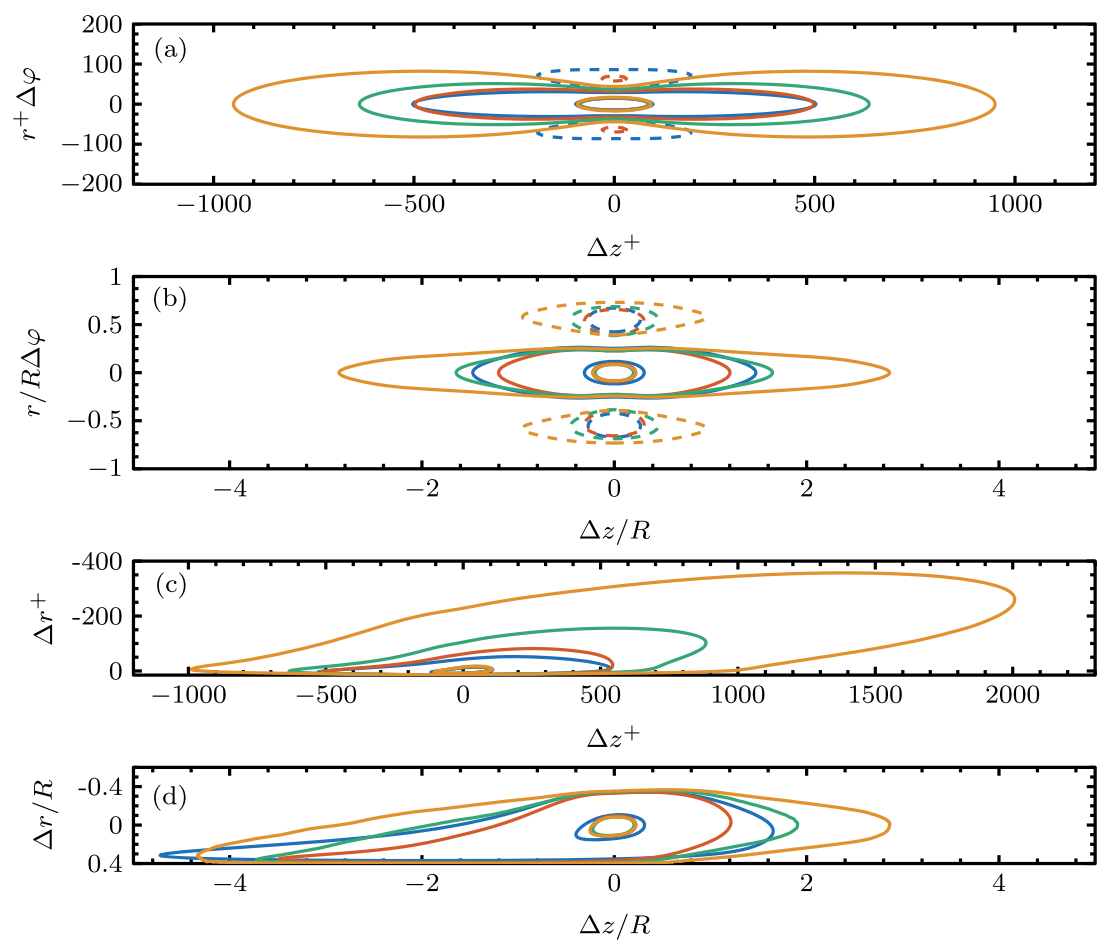

FIG. 10. Two-dimensional velocity correlations of the streamwise velocity $R_{z z}$ at different reference points. [(a) and (b)] $R_{z z}(\Delta z, \Delta \varphi, r)$. [(c) and (d)] $R_{z z}(\Delta z, \Delta r, r)$. [(e) and (f)] $R_{z z}(\Delta \varphi, \Delta r$, $r)$. [(a), (c), and (e)] $y^{+}=15$. [(b), (d), and (f) $] r / R=0.6$. Blue solid line, $R e_{\tau}$ = 180; orange solid line, $R e_{\tau}=360$; green solid line, $R e_{\tau}=720$; yellow solid line, $R e_{\tau}=1500$.
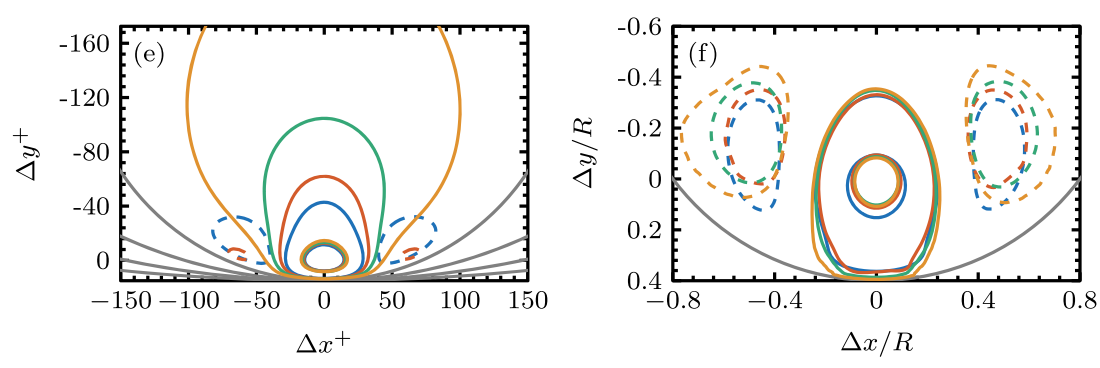


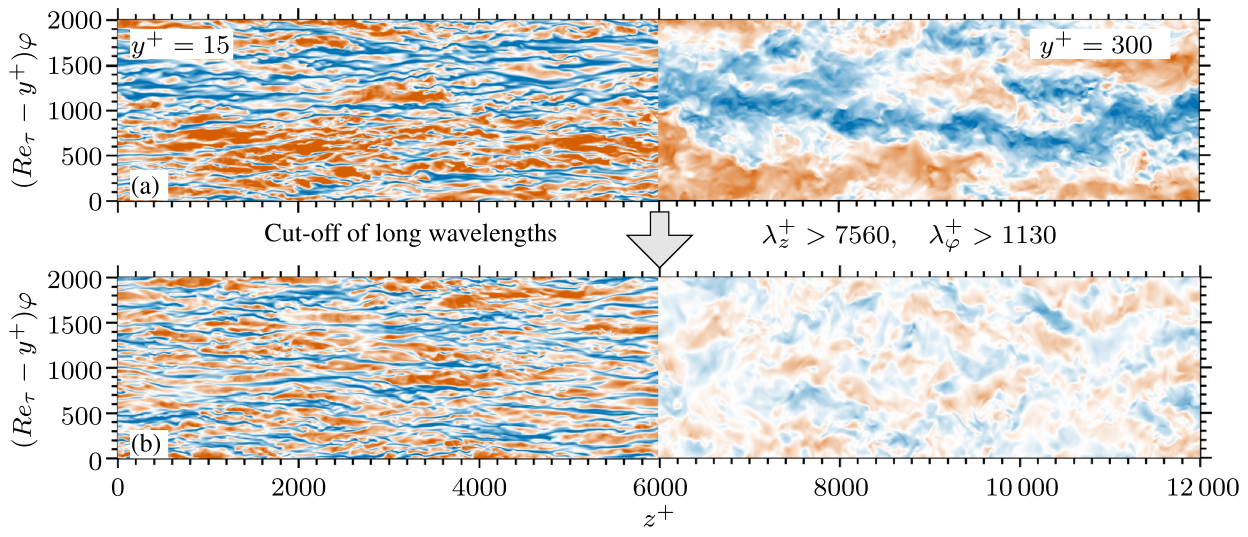

FIG. 11. Isocontours of streamwise velocity fluctuations $u_{z}^{\prime+}$ for $R e_{\tau}=1500$. (a) Before and (b) after spatial filtering. Left column plane at $y^{+}=15$, right column at $y^{+}=300$. Colormap is as in Fig. 9. extensions of coherent structures in the bulk flow are

$$
\begin{gathered}
l_{z, L S M} \times l_{\varphi, L S M} \times l_{r, L S M} \approx 3 R \times R \times 0.8 R, \\
l_{z, V L S M} \times l_{\varphi, V L S M} \times l_{r, V L S M} \approx 6 R \times R \times 0.8 R .
\end{gathered}
$$

\section{Interaction of coherent motions}

The failure of wall scaling of turbulent statistical quantities described in Sec. IV can be explained by the interaction of the turbulent coherent motions introduced in Secs. V A and $\mathrm{V} \mathrm{B}$. The $\left\langle u_{z}^{\prime} u_{z}^{\prime}\right\rangle$ peak is the signature of the most energetic species of structures, namely, the wall-layer streaks. The high wall-normal kurtosis value is the signature of the velocity spikes, as discussed in Sec. V A. Hoyas and Jiménez ${ }^{23}$ related the scaling failure of the streamwise Reynolds stress peak in turbulent channel flow to the interaction of largescale outer-flow motions with near-wall structures. Furthermore, Jiméne ${ }^{26}$ showed that the streamwise Reynolds stress scaling failure in the bulk region disappears, when energies related to structures longer than $\lambda_{x}=6 h$ and wider than $\lambda_{z}=h$ are removed from turbulent channel flow. This is subject to the condition that $x$ is the streamwise, $z$ is the spanwise coordinate, and $h$ is the channel half-height. A similar longwavelength cutoff filter, involving a constant filter length in wall units, is applied to an instantaneous flow-field realisation of the highest Reynolds number pipe-flow simulation, shown in Figs. 9(d) and 9(e). Figure 11(a) again shows the wall-layer streaks aligned coherently in packages of both rather highor low-speed streaks in the vicinity of the wall on the left side and a part of the outer-flow VLSM on the right side.
Figure 11(b) exhibits the same flow regions after the application of the long-wavelength cutoff filter to the instantaneous flow field, which was $F F$-transformed with respect to both homogeneous directions before. The filter lengths were chosen to match the full flow domain of the smallest Reynolds number simulation in wall units, yielding $\lambda_{z}^{+}=7560$ for the streamwise filter and $\lambda_{\varphi}^{+}=1130$ for the spanwise filter. After filtering the flow field, high and low velocity streaks appear to be distributed regularly alternating typical for low $R e$ flow. No regions carrying large amounts of energy are left in the bulk flow. Eliminating the kinetic energy contained in large streamand spanwise wavelengths from the picture effectively dissolves this conspicuous cluster formation of wall-layer streaks. This can be clearly seen by comparing the filtered with the unfiltered flow field at $y^{+}=15$ both shown in Fig. 11. It completely destroys the elongated large-scale motions at $y^{+}=300$, where most of the energy corresponds to large $\lambda$, as shown in Fig. 11.

After applying long-wavelength cutoff filters for streamand spanwise scales to all Reynolds number flows with a constant filter length in wall or bulk units, streamwise Reynolds stresses and skewness are computed. Figure 12 shows streamwise Reynolds stresses for the different Reynolds numbers involving long-wavelength filtered velocity fields with filter lengths constant in terms of wall units $\left[\lambda_{z}^{+}=5000, \lambda_{\varphi}^{+}=750\right.$, Fig. 12(a) $]$ and bulk units $\left[\lambda_{z}=6.5 R, \lambda_{\varphi}=0.975 R\right.$, Fig. 12(b)], as reported by Jiménez ${ }^{26}$ for turbulent channel flow. Note that the filter lengths were chosen to separate between the small-scale structures on the one hand and the large-scale motions on the other hand, as they are described in Sec. V B. In contrast to the unfiltered Reynolds stresses shown in Figs. 5(a)
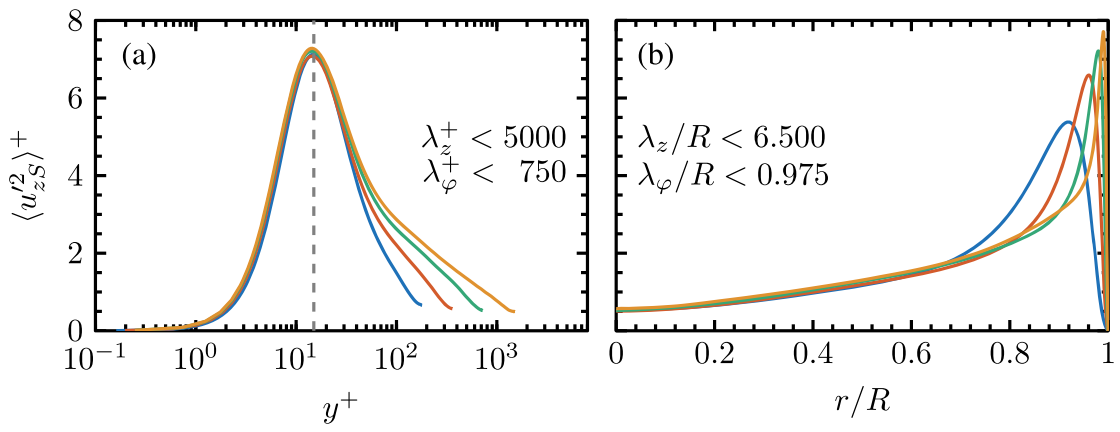

FIG. 12. Streamwise Reynolds stress computed from a long-wavelength filtered velocity signal. (a) Constant filter lengths in wall units, $\lambda_{z}^{+}<5000$, $\lambda_{\varphi}^{+}=750$. (b) Constant filter length in bulk units, $\lambda_{z}<6.5 R, \lambda_{\varphi}<0.975 R$. Blue solid line, $R e_{\tau}=180$; orange solid line, $R e_{\tau}=360$; green solid line, $R e_{\tau}$ $=720$; yellow solid line, $R e_{\tau}=1500$. 
and 5(b), the filtered Reynolds stresses now scale well in wall units in the near-wall [Fig. 12(a)] or bulk region [Fig. 12(b)] accordingly. The latter behaviour implies that the scaling failure of streamwise Reynolds stresses is caused by large-scale motions that become increasingly important with the Reynolds number.

In terms of streamwise skewness, the substitution of $u_{z}^{\prime}$ with the scale-decomposed velocity $u_{z}^{\prime}=u_{z L}^{\prime}+u_{z S}^{\prime}$ leads to the expression

$$
S\left(u_{z}\right)=\frac{1}{\left\langle u_{z}^{\prime 2}\right\rangle^{3 / 2}}\left(\left\langle u_{z L}^{\prime 3}\right\rangle+\left\langle u_{z L}^{\prime 2} u_{z S}^{\prime}\right\rangle+\left\langle u_{z L}^{\prime} u_{z S}^{\prime 2}\right\rangle+\left\langle u_{z S}^{\prime 3}\right\rangle\right) \text {. }
$$

Analysing experimental boundary-layer data, Mathis et al. ${ }^{10}$ showed that the Reynolds number dependency of the streamwise skewness profile is caused by the correlation term $\left\langle u_{z L}^{\prime} u_{z S}^{\prime 2}\right\rangle$ using a streamwise scale-decomposition with a filter length of $\lambda_{z}^{+}=7000$. They related the latter mentioned correlation term to the amplitude modulation term, ${ }^{9}$ which is responsible for the amplitude modulation of small scales by the large ones. In the Reynolds number regime of the current work, the streamwise scales are not sufficiently separated to apply a streamwise scale-decomposition only. Therefore, the contributions to the streamwise skewness in Eq. (18) are computed involving both a stream- and spanwise scale decomposition, where the small scales, contributing to $u_{z S}^{\prime}$, are no larger than $\lambda_{z}^{+}=5000$ and $\lambda_{\varphi}^{+}=750$, whereas the large scales, contributing to $u_{z L}^{\prime}$, exceed this limit in the stream- and/or in the spanwise direction. While the small-scale term $\left\langle u_{z S}^{\prime 3}\right\rangle /\left\langle u_{z}^{\prime 2}\right\rangle^{3 / 2}$ is the most dominant term in the vicinity of the wall, the correlation term $\left\langle u_{z L}^{\prime 2} u_{z S}^{\prime}\right\rangle /\left\langle u_{z}^{\prime 2}\right\rangle^{3 / 2}$ and the large-scale term $\left\langle u_{z L}^{\prime 3}\right\rangle /\left\langle u_{z}^{\prime 2}\right\rangle^{3 / 2}$ are close to zero near the wall and become important in the bulk flow region. The correlation term $\left\langle u_{z L}^{\prime} u_{z S}^{\prime 2}\right\rangle /\left\langle u_{z}^{\prime 2}\right\rangle^{3 / 2}$, presented in figure Fig. 13(a), exhibits a clear Reynolds number dependency. A reconstructed skewness

$$
S^{*}\left(u_{z}\right)=\frac{1}{\left\langle u_{z}^{\prime 2}\right\rangle^{3 / 2}}\left(\left\langle u_{z L}^{\prime 3}\right\rangle+\left\langle u_{z L}^{\prime 2} u_{z S}^{\prime}\right\rangle+\left\langle u_{z S}^{\prime 3}\right\rangle\right),
$$

where the last mentioned term is omitted, is shown in Fig. 13(b). Mathis et al. ${ }^{10}$ showed that the Reynolds number dependency disappears for the reconstructed skewness for turbulent boundary layer in the regime $2800 \leq R e_{\tau} \leq 19000$. Due to the limitations of the access to the very near-wall region in experimental investigations, their data were lacking any values below $y^{+} \approx 8$. In addition, the current pipe flow data acknowledge their findings for $180 \leq R e_{\tau} \leq 1500$ very close to the wall $y^{+}<15$, where no Reynolds number dependency of the reconstructed streamwise skewness could be determined. Further away from the wall where the scales in the current Reynolds number regime are not sufficiently separated, a scaling failure remains. Only between the two highest Reynolds numbers $R e_{\tau}=720$ and $R e_{\tau}=1500$ there is little variation of the reconstructed streamwise skewness profile throughout most of the flow domain. Higher Reynolds number data in terms of turbulent pipe flow are needed to obtain a Reynoldsnumber-independent reconstructed streamwise skewness profile.

Besides the interaction between streamwise large-scale outer flow and streamwise small-scale near-wall motions, the

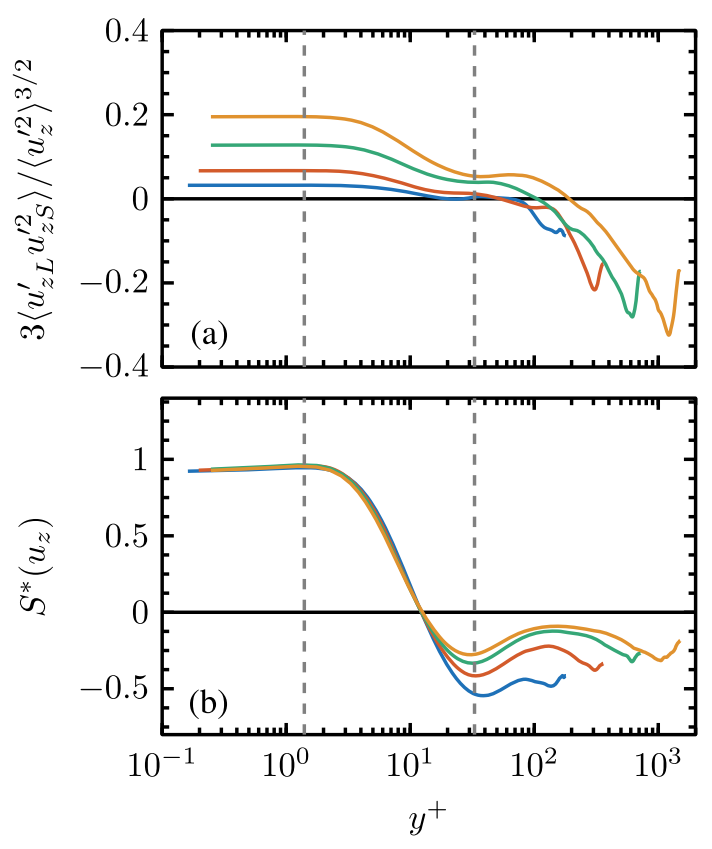

FIG. 13. (a) $\left\langle u_{z L}^{\prime} u_{z S}^{\prime 2}\right\rangle /\left\langle u_{z}^{\prime 2}\right\rangle^{3 / 2}$, contributions to the streamwise skewness, based on stream- and spanwise scale decomposition $u_{z}^{\prime}=u_{z L}^{\prime}+u_{z S}^{\prime}$, filter lengths $\lambda_{z}^{+}=5000, \lambda_{\varphi}^{+}=750$. (b) Reconstructed streamwise skewness $S^{*}\left(u_{z}\right)=\left(\left\langle u_{z L}^{\prime 3}\right\rangle+\left\langle u_{z L}^{\prime 2} u_{z S}^{\prime}\right\rangle+\left\langle u_{z S}^{\prime 3}\right\rangle\right) /\left\langle u_{z}^{\prime 2}\right\rangle^{3 / 2}$. Blue solid line, $R_{\tau}=180$; orange solid line, $R e_{\tau}=360$; green solid line, $R e_{\tau}=720$; yellow solid line, $R e_{\tau}=1500$.

interaction between the last mentioned and the wall-normal motions is to be elucidated. As discussed in Sec. V A, velocity spikes are predominantly found in regions of streamwise high momentum fluid and therefore in areas below highvelocity streaks. Conditionally averaged statistics underpin the conclusions from instantaneous flow-field realisations. Figure 14 shows the wall-normal flatness component obtained from regions, where the streamwise fluctuations are larger than zero on the one hand and smaller than zero on the other hand. While the wall-normal kurtosis value computed in low-speed streamwise momentum regions is relatively small in the vicinity of the wall, the value obtained from high-speed regions is much higher and more sensitive to the Reynolds number dependent failure of wall scaling. The

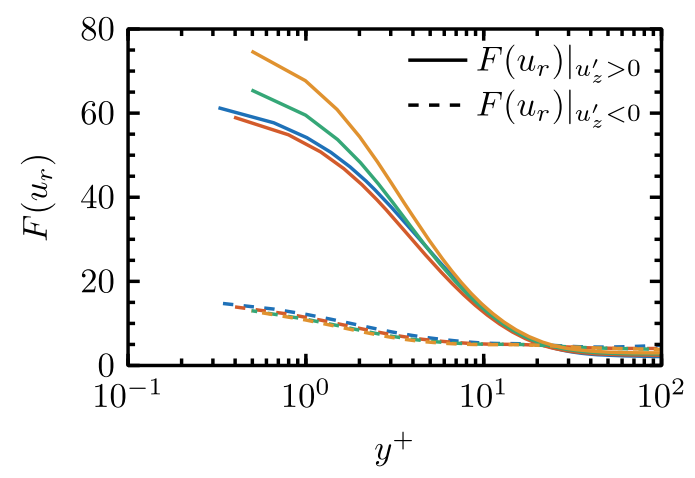

FIG. 14. Wall-normal flatness component $F\left(u_{r}\right)$, conditionally averagedbased on the streamwise velocity fluctuation. - $F\left(u_{r}\right)$, where $u_{z}^{\prime}>0$; - - , $F\left(u_{r}\right)$, where $u_{z}^{\prime}<0$. Blue solid line, $R e_{\tau}=180$; orange solid line, $R e_{\tau}=360$; green solid line, $R e_{\tau}=720$; yellow solid line, $R e_{\tau}=1500$. 
redistribution of velocity spikes appears to be strongly correlated with the redistribution of high-velocity streaks through outer-flow VLSM. Since regions of low streamwise momentum do not carry velocity spikes significantly, the kurtosis curve related to these regions is only marginally affected by the change of the Reynolds number, as the dashed lines in Fig. 14 indicate. The curve computed in high-speed regions, visualised by solid lines in Fig. 14, on the contrary, exhibits the same behaviour of scaling failure as the overall averaged wallnormal kurtosis, shown in Fig. 5. In summary, a similar scaling failure between the wall-normal kurtosis and the streamwise Reynolds stress in the vicinity of the wall, as discussed in Sec. IV, together with a high correlation between streaky structures of positive streamwise fluctuation and velocity spikes, underpinned by conditional averaged statistics (Fig. 14), was observed. This leads to the conclusion that the appearance of large-scale coherent motions in the bulk region of high Reynolds number flow is not only responsible for the scaling failure of the streamwise Reynolds stress and skewness profiles but also for the Reynolds number dependency in the wall-normal kurtosis component at the wall. Large- and verylarge-scale motions, which become increasingly active at high Reynolds numbers, interact with streamwise wall-layer streaks in terms of an alignment in packages. Since velocity spikes are strongly correlated with high-speed streaks, they are consequently rearranged in the same manner. These findings are in good agreement with the work of Lenaers et al., ${ }^{8}$ where the Reynolds number dependency of velocity spikes in plane channel flow for $180 \leq R e_{\tau} \leq 1000$ was related to the influence of large-scale motions with the aid of instantaneous flow-field realisations.

\section{CONCLUSION}

DNS of turbulent pipe flow within a flow domain of $L=42 R$ involving different Reynolds numbers and different wall-normal grid refinements was carried out and investigated in terms of convergence and scaling of turbulent highorder statistics and the interaction of underlying coherent structures.

While second-order moments like the Reynolds stresses were already fairly converged after an evaluation time of $\Delta \tau^{+}=10^{10}$, third- and fourth-order statistical moments, namely, wall-normal skewness and flatness in the vicinity of the wall needed to be integrated in time much longer to be statistically converged, i.e., $\Delta \tau^{+} \approx 2.6 \times 10^{11}$ and $\Delta \tau^{+} \approx 6.2$ $\times 10^{11}$, respectively. It was shown that the slow convergence is related to velocity spikes and their rare appearance.

The high wall-normal kurtosis value at the wall exhibited a high sensitivity with respect to the near-wall, wall-normal grid spacing caused by the asymptotic behaviour of the flatness when approaching the wall. In order to fulfil the no-slip boundary condition, wall-normal flatness was adapted from a steep gradient to a constant value in the very vicinity of the wall. To satisfy the no-slip condition in terms of wall-normal flatness, a minimal wall-normal grid spacing of $\Delta r^{+} \approx 0.3$ was found to be sufficient.

In terms of scaling of high-order statistics, logarithmic dependencies on the Reynolds number were observed for the streamwise Reynolds stress, where $R e_{\tau} \geq 360$, the streamwise skewness, and the wall-normal flatness for $R e_{\tau} \geq 360$ in the vicinity of the wall. The peak value of the streamwise Reynolds stress as well as the wall-normal flatness value at the wall computed from the current pipe-flow simulations exhibited discrepancies with respect to turbulent plane channel flow for the lowest Reynolds number $\left(R e_{\tau}=180\right)$. However, the values obtained from higher $R e_{\tau}$ simulations agreed fairly well. This behaviour is most probably caused by the flow geometry. At low Reynolds numbers, the wall structures are large in terms of bulk units and interact with the curvature of the pipe. At high Reynolds numbers, the influence of the curvature on the wall structures becomes increasingly negligible and the flow more similar to plane channel flow in the vicinity of the wall. In the bulk region, both streamwise Reynolds stresses and skewness scale well up to a Reynolds number of $R e_{\tau}=720$. For the highest Reynolds number simulation $\left(R e_{\tau}=1500\right)$, a sudden increase towards larger positive $\left(\left\langle u_{z}^{\prime} u_{z}^{\prime}\right\rangle\right)$ or negative $\left(S\left(u_{z}\right)\right)$ values appears, which the author relates to VLSM becoming important for this order of $R e_{\tau}$.

By analysing the turbulent coherent motions, the scaling failure of the streamwise Reynolds stress and streamwise skewness could be related to large-scale outer-flow motions. Deleting the influence of the large-scale contribution to the velocity field by means of scale-decomposition led to Reynolds-number-independent profiles of the streamwise Reynolds stress. In the vicinity of the wall, profiles collapsed with a filter length constant in wall units and in the bulk region with a filter length constant in bulk units. The Reynolds number dependency in the streamwise skewness in the very vicinity of the wall $\left(y^{+} \leq 15\right)$ was removed, when the contribution of the correlation term $\left\langle u_{z L}^{\prime} u_{z S}^{\prime 2}\right\rangle$, which is related to the amplitude modulation term, $9,10,42$ was omitted. Further away from the wall, the Reynolds number independency of the reconstructed skewness was not achieved, since the small and the large scales were not sufficiently separated at distances from the wall larger than $y^{+}=15$ and in a Reynolds number regime smaller than $R e_{\tau}=1500$.

In agreement with the turbulent plane channel study of Lenaers et al. ${ }^{8}$ the Reynolds number dependency of the wallnormal kurtosis could be related to the one of the streamwise Reynolds stress component, where outer-flow large-scale motions were found to penetrate the buffer layer. For higher Reynolds numbers, VLSMs were found to be mainly responsible for the alignment of wall-layer streaks in packages of high- and low-speed streaks, causing the failure of wall scaling in terms of the streamwise Reynolds stress peak. In addition, the strong correlation between high-speed streaks and velocity spikes, as indicated by conditional averaged kurtosis curves, was mainly responsible for the scaling failure of the wall-normal kurtosis in the vicinity of the wall. In summary, long elongated coherent motions in the bulk region of high Reynolds number turbulent flow penetrate the buffer layer thus influencing the alignment of wall-layer streaks and implicitly the one of velocity spikes. This leads to the scaling failure in terms of the wall-normal flatness component very close to the wall. While the values of the kurtosis level at the wall are similar between plane channel and pipe flow for large Reynolds numbers $\left(R e_{\tau} \approx 1000\right)$, where VLSMs 
play an important role, there is a large discrepancy at low Reynolds numbers. The wall-normal flatness value of $F\left(u_{r}\right) \approx 30$ at $y^{+}=1$ for $R e_{\tau}=180$ is higher than the channel flow value of $F(v) \approx 23.3$ at the same Reynolds number. This discrepancy in the wall-normal flatness is related to the different behaviour of wall-layer streaks in the two canonical cases, since the streaks are related to velocity spikes, as mentioned before. While the spanwise streak-spacing in plane channel flow exhibits values of $\lambda^{+} \approx 100$, the value obtained from pipe flow is somewhat larger $\lambda_{\varphi}^{+} \approx 120$. For higher Reynolds number, the impact of VLSMs, which are known to behave very similar in turbulent pipe and channel flows, ${ }^{17,38}$ probably led to higher kurtosis values in both cases and covered up the discrepancy.

\section{ACKNOWLEDGMENTS}

Computing resources on DLR's SCART cluster in Göttingen and computing time on SuperMUC provided by Leibniz Rechenzentrum under Grant No. pr62zu are gratefully acknowledged. D.F. also acknowledges partial financial support by the German Research Foundation (DFG) under Grant No. AV 20/3-1.

${ }^{1}$ C. Xu, Z. Zhang, J. M. J. den Toonder, and F. T. M. Nieuwstadt, "Origin of high kurtosis levels in the viscous sublayer. Direct numerical simulation and experiment," Phys. Fluids 8, 1938 (1996).

${ }^{2}$ F. Durst, J. Jovanovic, and J. Sender, "Detailed measurements of the near wall region of turbulent pipe flow," in Turbulent Shear Flows 9 (ASMEPublications-FED, 1995), Vol. 146, p. 225-240.

${ }^{3}$ J. M. J. den Toonder and F. T. M. Nieuwstadt, "Reynolds number effects in a turbulent pipe flow for low to moderate Re," Phys. Fluids 9, 3398-3409 (1997).

${ }^{4}$ J. G. M. Eggels, F. Unger, M. H. Weiss, J. Westerweel, R. J. Adrian, R. Friedrich, and F. T. M. Nieuwstadt, "Fully developed turbulent pipe flow: A comparison between direct numerical simulation and experiment," J. Fluid Mech. 268, 175 (1994).

${ }^{5} \mathrm{C}$. Wagner and R. Friedrich, "On the turbulence structure in solid and permeable pipes," Int. J. Heat Fluid Flow 19, 459-469 (1998).

${ }^{6} \mathrm{O}$. Shishkina and C. Wagner, "A fourth order finite volume scheme for turbulent flow simulations in cylindrical domains," Comput. Fluids 36, 484-497 (2007).

${ }^{7}$ B. J. Boersma, "Direct numerical simulation of turbulent pipe at high Reynolds numbers, velocity statistics and large scale motions," in Proceedings of the International Symposium on Turbulence and Shear Flow Phenomena, 2013.

${ }^{8}$ P. Lenaers, Q. Li, G. Brethouwer, P. Schlatter, and R. Örlü, "Rare backflow and extreme wall-normal velocity fluctuations in near-wall turbulence," Phys. Fluids 24, 035110 (2012).

${ }^{9}$ R. Mathis, N. Hutchins, and I. Marusic, "Large-scale amplitude modulation of the small-scale structures in turbulent boundary layers," J. Fluid Mech. 628, 311-337 (2009).

${ }^{10}$ R. Mathis, I. Marusic, N. Hutchins, and K. R. Sreenivasan, "The relationship between the velocity skewness and the amplitude modulation of the small scale by the large scale in turbulent boundary layers," Phys. Fluids $\mathbf{2 3}$, 121702 (2011).

${ }^{11}$ C. Chin, A. S. H. Ooi, I. Marusic, and H. M. Blackburn, "The influence of pipe length on turbulence statistics computed from direct numerical simulation data," Phys. Fluids 22, 115107 (2010).

${ }^{12}$ D. Feldmann, C. Bauer, and C. Wagner, "Computational domain length and Reynolds number effects on large-scale coherent motions in turbulent pipe flow," J. Turbul. (to be published).

${ }^{13}$ C. Wagner, "Dierkte numerische Simulation turbulenter Strömungen in einer Rohrerweiterung," Ph.D. dissertation (Technische Universität Münchenm, 1995).
${ }^{14}$ C. Wagner, T. J. Hüttl, and R. Friedrich, "Low-Reynolds-number effects derived from direct numerical simulations of turbulent pipe flow," Comput. Fluids 30, 581-590 (2001).

${ }^{15} \mathrm{D}$. Feldmann and C. Wagner, "Direct numerical simulation of fully developed turbulent and oscillatory pipe flows at $\operatorname{Re}_{\tau}=1440$," J. Turbul. 13, $1-28$ (2012).

${ }^{16}$ K. C. Kim and R. J. Adrian, "Very large-scale motion in the outer layer," Phys. Fluids 11, 417 (1999).

${ }^{17}$ J. P. Monty, N. Hutchins, H. C. H. Ng, I. Marusic, and M. S. Chong, "A comparison of turbulent pipe, channel and boundary layer flows," J. Fluid Mech. 632, 431-442 (2009).

${ }^{18}$ A. J. Chorin, "Numerical solution of the Navier-Stokes equations," Math. Comput. 22, 745-762 (1968).

${ }^{19} \mathrm{H}$. Tennekes and J. C. Wyngaard, "The intermittent small-scale structure of turbulence: Data-processing hazards," J. Fluid Mech. 55, 93-103 (1972).

${ }^{20} \mathrm{H}$. Tennekes and J. L. Lumley, A First Course in Turbulence (MIT Press, 1972).

${ }^{21}$ P. L. O'Neill, D. Nicolaides, D. Honnery, J. Soria et al., "Autocorrelation functions and the determination of integral length with reference to experimental and numerical data," in Proceedings of the 15th Australasian Fluid Mechanics Conference (The University of Sydney, 2004), Vol. 1, pp. 1-4.

${ }^{22}$ G. K. El Khoury, P. Schlatter, A. Noorani, P. F. Fischer, G. Brethouwer, and A. V. Johansson, "Direct numerical simulation of turbulent pipe flow at moderately high Reynolds numbers," Flow, Turbul. Combust. 91, 475-495 (2013).

${ }^{23} \mathrm{~S}$. Hoyas and J. Jiménez, "Scaling of the velocity fluctuations in turbulent channels up to $\operatorname{Re}_{\tau}=2003$," Phys. Fluids 18, 11702 (2006).

${ }^{24}$ M. Lee and R. D. Moser, "Direct numerical simulation of turbulent channel flow up to $\operatorname{Re}_{\tau}=5200$," J. Fluid Mech. 774, 395-415 (2015); e-print arXiv: 1410.7809 .

${ }^{25}$ J. Ahn, J. H. Lee, J.-H. Kang, and H. J. Sung, "Direct numerical simulation of a 30R long turbulent pipe flow at $\mathrm{Re}_{\tau}=3008$," Phys. Fluids 27, 065110 (2015)

${ }^{26}$ J. Jiménez, "Recent developments on wall-bounded turbulence," Rev. R. Acad. Cien. Serie A. Mat. 101, 187-203 (2007).

${ }^{27}$ S. K. Robinson, "Coherent motions in the turbulent boundary layer," Annu. Rev. Fluid Mech. 23, 601-639 (1991).

${ }^{28} \mathrm{~J}$. Kim, P. Moin, and R. Moser, "Turbulence statistics in fully developed channel flow at low Reynolds number," J. Fluid Mech. 177, 133-166 (1987).

${ }^{29}$ F. Unger, "Numerische Simulation turbulenter Rohrströmungen," Ph.D. dissertation (Technische Universität München, 1994).

${ }^{30}$ R. F. Blackwelder and H. Eckelmann, "Streamwise vortices associated with the bursting phenomenon," J. Fluid Mech. 94, 577 (1979).

${ }^{31}$ J. C. Del Álamo and J. Jiménez, "Spectra of the very large anisotropic scales in turbulent channels," Phys. Fluids 15, L41 (2003).

${ }^{32} \mathrm{H}$. Abe, H. Kawamura, and H. Choi, "Very large-scale structures and their effects on the wall shear-stress fluctuations in a turbulent channel flow up to $\operatorname{Re}_{\tau}=640$," J. Fluids Eng. 126, 835 (2004).

${ }^{33}$ M. Guala, S. E. Hommema, and R. J. Adrian, "Large-scale and very-largescale motions in turbulent pipe flow," J. Fluid Mech. 554, 521 (2006).

${ }^{34} \mathrm{~N}$. Hutchins and I. Marusic, "Evidence of very long meandering features in the logarithmic region of turbulent boundary layers," J. Fluid Mech. 579, 1 (2007).

${ }^{35}$ L. H. O. Hellström, A. Sinha, and A. J. Smits, "Visualizing the very-largescale motions in turbulent pipe flow," Phys. Fluids 23, 011703 (2011).

${ }^{36}$ L. H. O. Hellström and A. J. Smits, "The energetic motions in turbulent pipe flow," Phys. Fluids 26, 125102 (2014).

${ }^{37}$ A. J. Smits, B. J. McKeon, and I. Marusic, "High-Reynolds number wall turbulence," Annu. Rev. Fluid Mech. 43, 353-375 (2011).

${ }^{38}$ J. P. Monty, J. A. Stewart, R. C. Williams, and M. S. Chong, "Large-scale features in turbulent pipe and channel flows," J. Fluid Mech. 589, 147-156 (2007).

${ }^{39}$ J. Jiménez, "The largest scales of turbulent wall flows," CTR Annu. Res. Briefs 137, 54 (1998).

${ }^{40}$ J. C. Del Álamo, J. Jiménez, P. Zandonade, and R. D. Moser, "Scaling of the energy spectra of turbulent channels," J. Fluid Mech. 500, 135-144 (2004).

${ }^{41}$ J. Jiménez, "Cascades in wall-bounded turbulence," Annu. Rev. Fluid Mech. 44, 27-45 (2012).

${ }^{42} \mathrm{P}$. Schlatter and R. Örlü, "Quantifying the interaction between large and small scales in wall-bounded turbulent flows: A note of caution," Phys. Fluids 22, 051704 (2010). 